\title{
Single-Pixel Optical Fluctuation Analysis of Calcium Channel Function in Active Zones of Motor Nerve Terminals
}

\author{
Fujun Luo, ${ }^{1,2}$ Markus Dittrich, ${ }^{3}$ Joel R. Stiles, ${ }^{1,3,4}$ and Stephen D. Meriney ${ }^{1,2}$ \\ ${ }^{1}$ Department of Neuroscience, Center for Neuroscience, University of Pittsburgh, Pittsburgh, Pennsylvania 15260, ${ }^{2}$ Center for the Neural Basis of Cognition, \\ Pittsburgh, Pennsylvania 15213, ${ }^{3}$ National Resource for Biomedical Supercomputing, Pittsburgh Supercomputing Center, Pittsburgh, Pennsylvania 15213, \\ and ${ }^{4}$ Department of Biological Sciences, Carnegie Mellon University, Pittsburgh, Pennsylvania 15213
}

We used high-resolution fluorescence imaging and single-pixel optical fluctuation analysis to estimate the opening probability of individual voltage-gated calcium $\left(\mathrm{Ca}^{2+}\right)$ channels during an action potential and the number of such $\mathrm{Ca}^{2+}$ channels within active zones of frog neuromuscular junctions. Analysis revealed $\sim 36 \mathrm{Ca}^{2+}$ channels within each active zone, similar to the number of docked synaptic vesicles but far less than the total number of transmembrane particles reported based on freeze-fracture analysis $(\sim 200-250)$. The probability that each channel opened during an action potential was only $\sim 0.2$. These results suggest why each active zone averages only one quantal release event during every other action potential, despite a substantial number of docked vesicles. With sparse $\mathrm{Ca}^{2+} \mathrm{channels}$ and low opening probability, triggering of fusion for each vesicle is primarily controlled by $\mathrm{Ca}^{2+}$ influx through individual $\mathrm{Ca}^{2+}$ channels. In contrast, the entire synapse is highly reliable because it contains hundreds of active zones.

\section{Introduction}

Nerve terminal active zones contain highly organized components that underlie synaptic vesicle docking and fusion (Zhai and Bellen, 2004). Such organization is readily apparent in adult frog neuromuscular junctions (NMJs), in which electron microscopy has revealed hundreds of linear active zones, each arrayed with 200-250 intramembraneous particles and 25-40 closely associated synaptic vesicles (Heuser et al., 1979; Pawson et al., 1998; Harlow et al., 2001; Rizzoli and Betz, 2005). Previous investigators have hypothesized that these intramembraneous particles include the presynaptic $\mathrm{Ca}^{2+}$ channels that trigger vesicle fusion (Pumplin et al., 1981; Robitaille et al., 1990; Cohen et al., 1991). Because other channels and release machinery proteins are also present in the active zone (Robitaille et al., 1993), only some of the active zone particles observed in freeze-fracture replicas are expected to represent voltage-gated $\mathrm{Ca}^{2+}$ channels. Although the $\mathrm{NMJ}$ is known as a reliable synapse in which a single presynaptic action potential (AP) can trigger release of several hundred quanta (eliciting an action potential in the muscle) (Wood and Slater, 2001), each individual active zone operates as a low probability release site, averaging only one vesicle released following

Received March 18, 2011; revised May 18, 2011; accepted June 13, 2011

Author contributions: F.L., M.D., J.R.S., and S.D.M. designed research; F.L., M.D., and S.D.M. performed research; F.L., M.D., and J.R.S. analyzed data; F.L., M.D., J.R.S., and S.D.M. wrote the paper.

This work was supported by National Institutes of Health Grant R01 NS043396 (S.D.M.), R01 GM068630, P41 RR06009, and P20 GM065805 (J.R.S.), and F32 GM08347301 (M.D.) and National Science Foundation Research Grant 0844604 (S.D.M.). We thank Greg Hood for writing the image alignment routine, Robert Poage for assistance with some early experiments, and Soyoun Cho and Kate Cosgrove for their comments on previous versions of this manuscript.

Correspondence should be addressed to Dr. Stephen D. Meriney, Department of Neuroscience, A210 Langley Hall, University of Pittsburgh, Pittsburgh, PA 15260. E-mail: meriney@pitt.edu.

DOI:10.1523/JNEUROSCI.1394-11.2011

Copyright $\odot 2011$ the authors $\quad 0270-6474 / 11 / 3111268-14 \$ 15.00 / 0$ every other action potential (Katz, 1969; Katz and Miledi, 1979; Poage and Meriney, 2002).

Despite $>60$ years of study, many important questions persist regarding $\mathrm{Ca}^{2+}$-dependent transmitter release. For example, it is not known how many voltage-gated $\mathrm{Ca}^{2+}$ channels are positioned in an active zone or what is the in situ probability $(p)$ that they will open during an action potential [which is distinct from the channel open probability $\left(P_{\mathrm{o}}\right)$ traditionally measured during long depolarizing steps]. Answers to both of these questions will provide important constraints on models of $\mathrm{Ca}^{2+}$-triggered vesicle fusion, including the stoichiometric and spatial relationships between $\mathrm{Ca}^{2+}$ channels and docked vesicles. To address these questions, we developed single-pixel optical fluctuation analysis (SPOFA) and applied it to action potential-evoked $\mathrm{Ca}^{2+}$ indicator fluorescence measurements in frog motor nerve terminals.

Variance analysis of $\mathrm{Ca}^{2+}$ fluorescence signals has been applied previously to small neuronal compartments and used to reveal stochastic openings of $\mathrm{Ca}^{2+}$ channels in presynaptic boutons and postsynaptic spines (Frenguelli and Malinow 1996; Sabatini and Svoboda, 2000) (but see Brenowitz and Regehr, 2007). In addition, we have previously described stochastic $\mathrm{Ca}^{2+}$ signals arising from frog NMJ active zones (Wachman et al., 2004). We now extend this approach to automated acquisition and analysis of signal variance at the level of single pixels overlying portions of active zones. In doing so, we characterize the spatial resolution of data acquisition, the methods used for selection of active zone pixels, and the possible contribution of single-channel current variability to the measured variability. We demonstrate optical resolution at subactive zone dimensions, show that our results are primarily independent of pixel selection criteria, and also consider the effect of additional sources of variation on our results via Monte Carlo (MCell) simulations. Based on our findings, we conclude that relatively few $\mathrm{Ca}^{2+}$ channels line each active zone of 
A

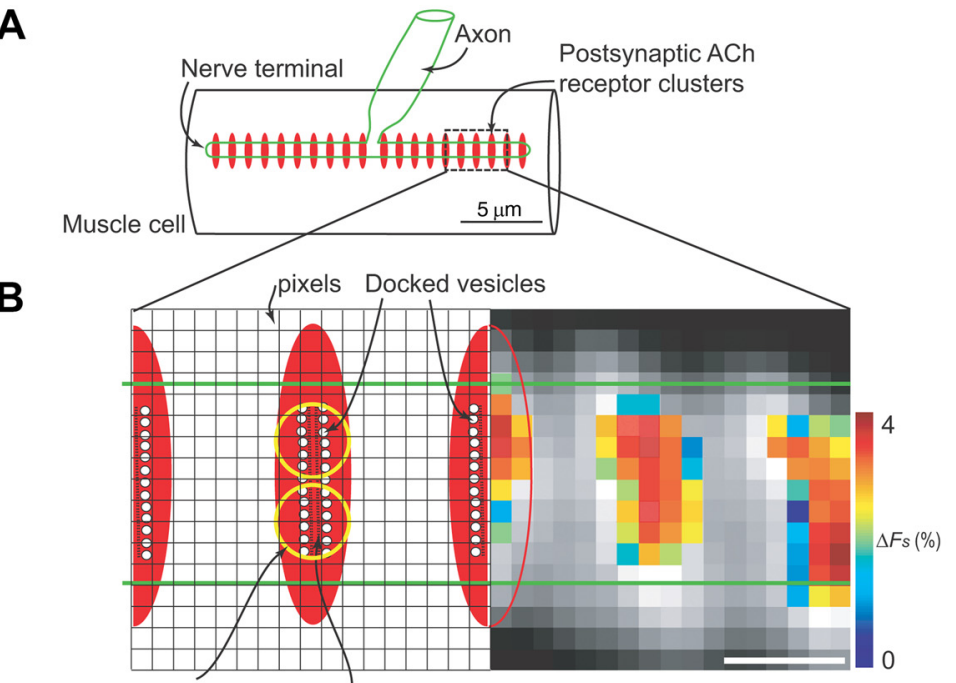

Estimated optical Active zone proteins resolution
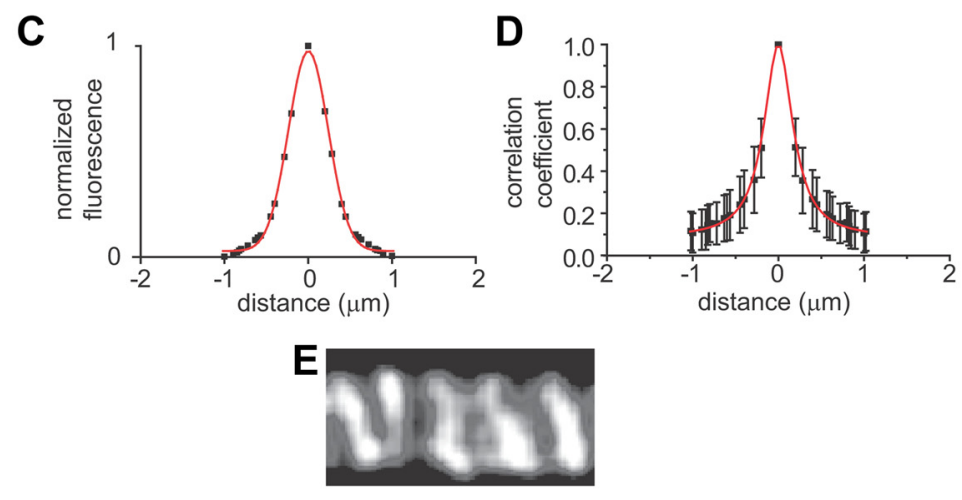

$\mathbf{F}$
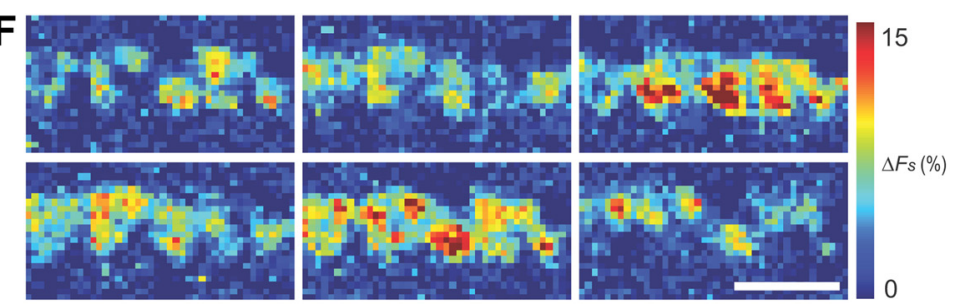

Figure 1. Pixel and optical resolution for calcium imaging in the adult frog neuromuscular junction. $A$, Diagram of the adult frog neuromuscular junction. $\boldsymbol{B}$, Magnified active zones (boxed region in $\boldsymbol{A}$ ) with highly organized active zone proteins, which include voltage-gated $\mathrm{Ca}^{2+}$ channels, and docked synaptic vesicles. The estimated relationship between active zone dimensions, pixel size, and optical resolution are also shown (left). For comparison, a composite image that includes fluorescent staining of postsynaptic acetylcholine receptors (background, grayscale) overlaid with $\mathrm{Ca}^{2+}$-evoked fluorescent signals after nerve stimulation (foreground, false color) is shown on the right. Scale bar, $1 \mu \mathrm{m}$. C, Experimentally measured point spread function of the optical system from a fluorescent bead with diameter of $0.02 \mu \mathrm{m}$. The Gaussian fit (red) has a width at half-amplitude of $0.45 \mu \mathrm{m}$. We thus estimated that approximately half the length of a single active zone can be resolved optically. $\boldsymbol{D}$, Correlation coefficient of neighboring pixels that detected AP-evoked fluorescence signals after repeated low-frequency nerve stimulus tightly depends on the distance between pixels. The Lorentzian fit (red) has a width at half-amplitude of $0.41 \mu \mathrm{m}$ (points represent mean $\pm S D ; n=$ 4361 pixels). These data are fit best with a Lorentzian function, rather than a Gaussian, likely because of the presence of some signal contributing from neighboring sites, especially at the tails of the distribution. $\boldsymbol{E}$, Predicted active zone regions of a representative frog motor nerve terminal identified by labeling the postsynaptic acetylcholine receptor clusters with Alexa Fluor $594-\alpha$ bungarotoxin. $\boldsymbol{F}$, Representative difference images showed a large trial-to-trial variability in the spatial distribution of $\mathrm{Ca}^{2+}$ entry evoked by single APs. Scale bar, $2 \mu \mathrm{m}$ (applies to $\boldsymbol{E}, \boldsymbol{F}$ ).

frog NMJs and that each action potential normally opens only a small fraction of such channels. These results lead us to hypothesize that action potential-evoked vesicle fusion at this synapse is likely triggered by the $\mathrm{Ca}^{2+}$ ion flux through one or very few $\mathrm{Ca}^{2+}$ channels.

\section{Materials and Methods}

Dye loading and staining of cutaneous pectoris nerve-muscle preparations Adult frogs (Rana pipiens) were decapitated and pithed after anesthesia in $0.1 \%$ tricaine methane sulfonate. Cutaneous pectoris muscles were dissected bilaterally and bathed in normal frog Ringer's solution (NFR) (in mm: $116 \mathrm{NaCl}, 2 \mathrm{KCl}, 1.8 \mathrm{CaCl}_{2}, 1 \mathrm{MgCl}_{2}$, and 5 HEPES, pH 7.4). The nerve was cut near its entrance into the muscle, and the cut end was drawn into a Vaseline well containing $30 \mathrm{~mm}$ Calcium Green-1 (3000 molecular weight dextran conjugate; Invitrogen) dissolved in distilled water. After $7-8 \mathrm{~h}$ of dye loading at room temperature, the preparation was rinsed in NFR and stored at $4^{\circ} \mathrm{C}$ for $2-3 \mathrm{~h}$. For stimulation and imaging, preparations were pinned over an elevated Sylgard (Dow Corning) platform in a $35 \mathrm{~mm}$ dish mounted on the microscope stage. The nerve was drawn into a suction electrode, and stimulation threshold was determined by observation of muscle twitch. Postsynaptic acetylcholine receptors then were blocked and labeled using $2 \mu \mathrm{g} / \mathrm{ml}$ Alexa Fluor 594-conjugated $\alpha$-bungarotoxin $(\alpha$-BTX) for 10 min. $\alpha$-BTX staining was used to locate and focus the postsynaptic receptor bands, which are directly opposed to the presynaptic active zones, and to evaluate possible $z$-axis drift over the course of data collection. Superficial nerve terminals were chosen for study, and most lay in a single focal plane as judged by $\alpha$-BTX staining. Except as noted, all $\mathrm{Ca}^{2+}$ imaging was performed in NFR containing $10 \mu \mathrm{M}$ curare to prevent nerve-evoked muscle contractions not completely blocked by $\alpha$-BTX.

\section{Calcium imaging and nerve stimulation protocol}

Images were collected at $0.5 \mathrm{~Hz}$ using a $1 \mathrm{~ms}$ laser illumination time. An acousto-optic tunable filter (ChromoDynamics) was used to select wavelengths and gate the laser with submillisecond time resolution (krypton-argon laser; Innova 70 Spectrum; Coherent). The laser was fiber coupled to the epi-illumination port of an upright fluorescence microscope (Olympus BX61WI) equipped with a longworking-distance water-immersion objective (100×, 1.0 NA; Lumplan/FL IR; Olympus). Calcium Green-1 was excited at $488 \mathrm{~nm}$, and emitted light was collected through a $530 \pm 20$ $n m$ filter. Alexa Fluor $594-\alpha$-BTX was excited at $567 \mathrm{~nm}$, and emitted light was collected using a $620 \pm 30 \mathrm{~nm}$ filter. Images were recorded on one of two cooled, back-thinned CCD cameras (LN1300B by Roper Scientific; or Ixon DV887 by Andor), which provided the high sensitivity and low noise necessary for imaging acquisition during $1 \mathrm{~ms}$ illumination times. Pixel size was $200 \mathrm{~nm}$ (LN1300B) or $160 \mathrm{~nm}$ (Ixon DV887). Analysis of data from either camera gave similar results. In comparing theoretical Rayleigh's and FWHM criteria (based on the optics within our microscope and the excitation wavelength of the fluorescent dye), our axial resolution can be no better than between 864 and $976 \mathrm{~nm}$. The lateral resolution of the microscope was experimentally 
determined by measuring the point-spread function (PSF) imaged using $20 \mathrm{~nm}$ fluorescent beads (after Gaussian fit, width at half-maximum amplitude of $450 \mathrm{~nm}$ ) (Fig. 1C). Furthermore, a plot of the crosscorrelation analysis of action potential-evoked fluorescent signals in the nerve terminal detected by adjacent pixels revealed a width at half-maximum amplitude of $410 \mathrm{~nm}$ (Fig. $1 D$ ). These data indicate that, as a result of our brief $1 \mathrm{~ms}$ acquisition window, signals sampled by a given pixel derive mainly from channels underlying this pixel. This distribution is best fit by a Lorentzian function because there is some optical signal that appears to contribute to the "tails" of this distribution. The signal sampled by one pixel does not derive exclusively from a single point source (a single open $\mathrm{Ca}^{2+}$ channel centered below the pixel), but rather there are likely neighboring sources that contribute some elevated signal because we predict that several spatially distributed $\mathrm{Ca}^{2+}$ channels likely open within one active zone after each action potential. Given the anatomical organization of active zones and considering our $1 \mathrm{~ms}$ illumination timed with action potential invasion (which limits time for diffusion), a pixel that is positioned over one active zone will likely not sample significant signal derived from calcium channel flux originating within neighboring active zones. However, within the sampling region detected by one pixel, we do not have a good method for determining the heterogeneity in contribution that might arise from different $\mathrm{Ca}^{2+}$ channels that might be at slightly different distances from the center of one pixel.

During acquisition, images were collected in sets of 20 . Within each set, the first 10 were acquired without nerve stimulation to obtain resting (background) fluorescence, whereas each of the second 10 followed nerve stimulation at $5 \times$ threshold to obtain action potential-dependent fluorescence. A delay of $1.5 \mathrm{~ms}$ between stimulation and illumination was used to account for nerve conduction delay in this preparation. In most experiments, 10 sets of trials were performed sequentially to obtain a total of 100 background images interleaved with 100 images after stimulation. Between sets, we confirmed or adjusted focus based on $\alpha$-BTX staining (which allowed a characterization of active zone distribution and focal plane) and discarded image sets from analysis if they showed noticeable $z$-axis drift or significant changes in average background fluorescence. In some experiments, the preparation subsequently was exposed to the potassium channel blocker 3,4-diaminopyridine (DAP) (5 $\mu \mathrm{M}$ for $30 \mathrm{~min}$ ), and another 10 sets of images were obtained in the continued presence of DAP. Because there is active propagation of action potentials along the length of motor nerve terminals (Katz and Miledi, 1965, 1968; Braun and Schmidt, 1966; Mallart, 1984), DAP treatment will block presynaptic potassium channels, prolonging the duration of the action potential (Kirsch and Narahashi, 1978; Durant and Marshall, 1980 ), without altering propagation within the nerve terminal. As such, this treatment will indirectly increases the probability $(p)$ that $\mathrm{Ca}^{2+}$ channels open after nerve stimulation but leave the number of channels (n) unchanged. This differential effect allows subsequent analysis of fluorescence fluctuations across repeated trials to determine $p$ and $n$ on a per pixel basis (see below).

\section{Image registration and analysis}

Before analysis, all images collected from individual terminals were registered to the first image to correct for slight fluctuations in the $x-y$ (or lateral) position of the preparation during data collection. Registration software was written at the National Resource for Biomedical Supercomputing (www.nrbsc.org) at the Pittsburgh Supercomputing Center. To avoid aligning on background features, we sampled the most prominent aspects of the background (including any artifacts) and subtracted this background from the images. We then used the raw calcium-dependent fluorescence to create a mask that represented the detailed shape of the nerve terminal in each image. With this approach, we used customwritten nonlinear registration software to warp each image in a time series to the first image of that series. Although the software was capable of generalized rotational and nonlinear corrections, virtually all of the adjustments necessary for these experiments were simple interpolated translational movements of one pixel or less. Stimulusdependent changes in fluorescence intensity were small (typically
Table 1. The impact of pixel selection on average values of $p$ and $n$

\begin{tabular}{lll}
\hline Pixel selection criteria & $p$ & $n$ \\
\hline$p<10^{-2}$ & $0.26 \pm 0.11$ & $12.1 \pm 2.8$ \\
$p<10^{-3}$ & $0.26 \pm 0.11$ & $14.1 \pm 3.0$ \\
$p<10^{-6}$ & $0.25 \pm 0.10$ & $17.7 \pm 4.2$ \\
$p<10^{-9 *}$ & $0.24 \pm 0.10$ & $18.4 \pm 1.2$ \\
SD criterion & $0.24 \pm 0.11$ & $18.3 \pm 4.8$ \\
\hline
\end{tabular}

Pixels were selected for analysis based on a Student's t test ( $p$ values) or our SD criterion (see Materials and Methods). Values shown are mean $\pm S D$ for four or three (asterisk) nerve terminals, and the values for $p$ correspond to the control (pre-DAP treatment) condition.

3-4\% above background; see Results), and so the registration step brought the shape of the nerve terminal into alignment without being confounded by large and variable changes in signal strength within regions of nerve terminals. Analysis of registered images was performed using MATLAB. To create difference images showing stimulus-dependent fluorescence changes $\left(\Delta F_{s}\right)$ for each pixel, we calculated the average background fluorescence of each pixel $\left(\bar{f}_{b}\right)$, subtracted it from the fluorescence $\left(f_{s}\right)$ obtained after a stimulation trial, and then normalized the resulting differences to $\bar{f}_{b}$, i.e., per pixel $\Delta f_{s}=\frac{f_{s}-\bar{f}_{b}}{\bar{f}_{b}}$. Resulting difference images were displayed in pseudocolor. For subsequent calculation of $n$ and $p$ (see below), analysis for each pixel also included calculation of the background variance $\left(\sigma_{b}^{2}\right)$, the mean fluorescence $\left(\bar{f}_{s}\right)$ and variance $\left(\sigma_{s}^{2}\right)$ after nerve stimulation, and the mean fluorescence $\left(\bar{f}_{D s}\right)$ and variance $\left(\sigma_{D s}^{2}\right)$ after stimulation combined with DAP treatment. Within image sets from individual terminals, the variance of background fluorescence arises from dark noise and photon shot noise and, under our experimental conditions, was dominated by Gaussian shot noise. Conversely, background photoelectron counts from different visualized nerve terminals varied according to differences in geometry and dye loading and ranged from 1500 to 6000 per pixel. With resting photoelectron counts this high and stimulated fluorescence changes that were $<15 \%$, the contribution of differences in shot noise between resting and stimulated measurements to our final results would have been very small $(<1 \%)$ and were thus ignored.

\section{Determination of $\mathrm{n}$ and $\mathrm{p}$}

Over the course of repeated stimulation trials, the mean fluorescence intensity $\bar{f}_{s}$ is expected to exceed the mean background level $\bar{f}_{b}$ for pixels that overlie a subregion of an active zone and periodically detect $\mathrm{Ca}^{2+}$ influx through one or more open $\mathrm{Ca}^{2+}$ channels. We limited our subsequent analysis to those pixels within active zone regions that reported significant action potential-evoked fluorescence signals under control conditions. We evaluated a range of criteria for significance to rule out sampling effects on our results as much as possible. For most of our analyses, we settled on using the criterion that the stimulus-dependent mean fluorescence $\left(\Delta f_{s ;}\right.$ see below) be greater than the SD of background fluorescence $\sigma_{b}$ (SD criterion). We demonstrated that the choice of selection criterion had little or no effect on our estimates of $n$ and $p$ by comparing results using this criterion with a Student's $t$ test using different levels of significance (Table 1) (see Figs. 6 and 7).

We calculated $n$ and $p$ for selected pixels using two measurable ratios, $R_{F}$ and $R_{\mathrm{CV}}$ (Sabatini and Svoboda, 2000). The mean of the stimulusdependent fluorescence signals before and after DAP treatment are as follows:

$$
\begin{aligned}
\Delta F_{s} & =\frac{\bar{f}_{s}-\bar{f}_{b}}{\bar{f}_{b}} \times 100 \%, \\
\Delta F_{D s} & =\frac{\bar{f}_{D s}-\bar{f}_{b}}{\bar{f}_{b}} \times 100 \% .
\end{aligned}
$$

We define $R_{F}$ as the ratio of stimulus-dependent fluorescence signals before and after DAP treatment: 


$$
R_{F}=\frac{\Delta F_{s}}{\Delta F_{D s}}
$$

Similarly, the coefficient of variation (CV) in the stimulus-dependent fluorescence signals before and after DAP treatment are as follows:

$$
\begin{aligned}
\mathrm{CV}_{s} & =\frac{\sqrt{\sigma_{s}^{2}-\sigma_{b}^{2}}}{\bar{f}_{s}-\bar{f}_{b}} \\
\mathrm{CV}_{D s} & =\frac{\sqrt{\sigma_{D s}^{2}-\sigma_{b}^{2}}}{\bar{f}_{D s}-\bar{f}_{b}}
\end{aligned}
$$

$R_{\mathrm{CV}}$ is defined as follows:

$$
R_{\mathrm{CV}}=\mathrm{CV}_{s}^{2} / \mathrm{CV}_{D s}^{2}=\frac{\sigma_{s}^{2}-\sigma_{b}^{2}}{\left(\bar{f}_{s}-\bar{f}_{b}\right)^{2}} / \frac{\sigma_{D s}^{2}-\sigma_{b}^{2}}{\left(\bar{f}_{D s}-\bar{f}_{b}\right)^{2}} .
$$

To calculate $n$ and $p$ from the experimental $R_{F}$ (Eq. 1c) and $R_{\mathrm{CV}}$ (Eq. 2c) values, we assume that each $\mathrm{Ca}^{2+}$ channel acts independently and opens during an action potential with probability $p$ before DAP treatment and $p_{D}$ after DAP treatment. The corresponding probabilities of remaining closed are $(1-p)$ and $\left(1-p_{D}\right)$, respectively. Under each condition, the number of channels that open from trial to trial is expected to follow a binomial distribution. From standard binomial theory, in a sub-active zone region containing $n$ channels, the average number that will open $(\mu)$ before DAP treatment is given by:

$$
\mu=n p .
$$

Assuming a linear detection system (see Results), the mean fluorescence arising from $\mathrm{Ca}^{2+}$ entering through open channels $\left(\Delta F_{s}\right.$; Eq. 1a) will be proportional to $\mu$, thus

$$
\Delta F_{s} \propto \mu=n p .
$$

Similarly, after DAP treatment, the mean fluorescence arising from entering $\mathrm{Ca}^{2+}\left(\triangle F D_{s}\right.$; Eq. 1b) will be proportional to the average number that will open $\mu_{D}$ after DAP treatment:

$$
\Delta F_{D s} \propto \mu_{D}=n p_{D} .
$$

The mean stimulus-dependent fluorescence signals before and after DAP treatment should be governed by the number of $\mathrm{Ca}^{2+}$ channels opened by an action potential.

From Equations 1c, 5, and 6, we now have

$$
R_{F}=\frac{\Delta F_{s}}{\Delta F_{D s}}=\frac{n p}{n p_{D}}=\frac{p}{p_{D}},
$$

where we assume that DAP treatment does not affect the number of functional $\mathrm{Ca}^{2+}$ channels $n$ as well as the amount of $\mathrm{Ca}^{2+}$ flux through single open channels (see Results).

Again from standard binomial theory, the predicted variance for channel opening before DAP treatment $\sigma^{2}$ is

$$
\sigma^{2}=n p(1-p)
$$

The $\mathrm{SD}(\sigma)$ is thus:

$$
\sigma=\sqrt{n p(1-p)} .
$$

Using Equations 3 and 8, the CV in the number of open channels is

$$
\begin{gathered}
\mathrm{CV}=\frac{\sigma}{\mu}=\sqrt{\frac{1-p}{n p} .} \\
\mathrm{CV}^{2}=\frac{1-p}{n p} .
\end{gathered}
$$

If all of the variance in measured stimulus-dependent fluorescence arises from the random openings of channels from trial to trial, then from Equations 2a and 7,

$$
\left(\sigma_{s}^{2}-\sigma_{b}^{2}\right) \propto \sigma^{2}=n p(1-p),
$$

and from Equations $2 \mathrm{a}$ and $9 \mathrm{~b}$,

$$
\mathrm{CV}_{s}^{2} \propto \mathrm{CV}^{2}=\frac{1-p}{n p}
$$

Similarly, after DAP treatment,

$$
\mathrm{CV}_{D s}^{2} \propto \frac{1-p_{D}}{n p_{D}}
$$

Therefore, from Equations 2c, 11, and 12, we have

$$
R_{\mathrm{CV}} \equiv \mathrm{CV}_{s}^{2} / \mathrm{CV}_{D s}^{2}=\frac{1-p}{n p} / \frac{1-p_{D}}{n p_{D}}=\frac{1-p}{p} / \frac{1-p_{D}}{p_{D}}
$$

where again we assume that DAP treatment does not affect the number of $\mathrm{Ca}^{2+}$ channels $n$ as well as the amount of $\mathrm{Ca}^{2+}$ flux through single open channels.

In Equations 6 and 13, we have the two measured ratios $R_{F}$ and $R_{\mathrm{CV}}$, as well as the two unknown probabilities $p$ and $p_{D}$. Rearranging Equation 6 for $p_{D}$ yields the following:

$$
p_{D}=\frac{p}{R_{F}}
$$

After using Equation 14 to substitute for $p_{D}$ in Equation 13, algebraic rearrangement now provides an expression for $p$ that includes only the measured ratios $R_{F}$ and $R_{\mathrm{CV}}$ :

$$
p=\frac{R_{\mathrm{CV}} R_{F}-1}{R_{\mathrm{CV}}-1}
$$

Equation 15 was therefore used to calculate $p$, and Equation 14 was used to calculate $p_{D}$. Finally, a rearranged form of Equation 11 was used to estimate $n$ :

$$
n=\frac{1-p}{p \mathrm{CV}_{s}^{2}}
$$

\section{Computational modeling of action-potential-triggered \\ $\mathrm{Ca}^{2+}$ fluorescence}

Generation of action potentials. Action potentials were generated with a standard Hodgkin-Huxley model using the NEURON simulation environment (release 7.1.) (Hines and Carnevale, 1997; Carnevale and Hines, 2006). The parameters for the Hodgkin-Huxley model were chosen based on two criteria for the resulting action potentials. First, because adult motor nerve terminals are not accessible to direct recordings of action potential shape, we based our assumptions for AP shape on experimentally measured synaptic action potential waveforms from embryonic cultured motor nerve terminals, which are very narrow. Second, when used within our computational model of action-potentialtriggered $\mathrm{Ca}^{2+}$ influx (see below), we aimed for a $\mathrm{Ca}^{2+}$ channel open probability $p$ under control conditions of $\sim 0.2$ (AP1, 0.18; AP2, 0.20). In choosing modeled APs for our analysis, we sought to use one AP shape that was very similar to the recorded AP from embryonic cultured motor nerve terminals (AP2). Because we cannot be sure of the exact shape of the $\mathrm{AP}$ at adult frog motor nerve terminals and to be more rigorous in our analysis, we also considered a second AP (AP1) with a distinctly different AP shape but still subject to the same constraints on open probability $p$ for $\mathrm{Ca}^{2+}$ channels. The two thus generated action potential waveforms AP1 and AP2 are depicted in Figure $3 A$. Figure $3 B$ shows both action potentials together with an experimentally recorded action potential recorded from cultured embryonic Xenopus nerve-muscle synapses (Pattillo et al., 2001). 
The effect of DAP on action potential shape was modeled by reducing the $g_{k, \max }$ value of the potassium gating particles in the Hodgkin-Huxley model. The resulting broadening of the action potentials (see Fig. $3 A$ ) led to an increase in the $\mathrm{Ca}^{2+}$ channel open probability $p$, and $g_{k \text {, max }}$ was chosen to yield a $p$ value of $\sim 0.6$ (AP1, 0.56; AP2, 0.65). It is important to point out that the values we chose for $p$ were arbitrary (but very close to experimental observations) because our computational validation procedure only seeks to establish the ability of SPOFA to estimate the values of $p$ and $n$ regardless of their actual values.

Monte Carlo simulations of action-potential-triggered $\mathrm{Ca}^{2+}$ influx. To test whether SPOFA is able to estimate $p$ and $n$ from measured fluorescence data, we developed a computation model of action-potential-triggered $\mathrm{Ca}^{2+}$ influx through voltage-gated $\mathrm{Ca}^{2+}$ channels. All simulations were performed with MCell version 3 (Monte Carlo Cell) (Stiles et al., 1996; Stiles and Bartol, 2001; Kerr et al., 2008) and were typically run on a desktop computer running the Linux operating system. MCell allows one to perform continuous time stochastic simulations of action-potential-triggered $\mathrm{Ca}^{2+}$ channel opening and subsequent $\mathrm{Ca}^{2+}$ ion flux into the presynaptic nerve terminal through the channel. We created several simulation models each containing a fixed number of $\mathrm{Ca}^{2+}$ channels $(1,10,15,20,30$, and $40 \mathrm{Ca}^{2+}$ channels, respectively) embedded in a membrane. During each simulation, $\mathrm{Ca}^{2+}$ channels opened stochastically driven by the action potential and permeated $\mathrm{Ca}^{2+}$ ions according to the kinetic scheme depicted in Figure $3 C$ consisting of three closed states, one open state, and voltage-dependent rates between states. The driving force $k_{\text {flux }}$ for $\mathrm{Ca}^{2+}$ through the open channels is shown in the bottom of Figure $3 \mathrm{~A}$. Rates were chosen such that the model reproduced the experimentally known single-channel conductance (2.6 pS in $2 \mathrm{~mm}$ extracellular $\mathrm{Ca}^{2+}$ ) (Church and Stanley, 1996) and the experimentally measured whole-cell currents (see Fig. 3B) and Pattillo et al. (2001). For each model containing a given number of $\mathrm{Ca}^{2+}$ channels and action potential AP1 and AP2, we ran 10,000 simulations under control and DAP conditions. The resulting $\mathrm{Ca}^{2+}$ currents were recorded and then used as a proxy for fluorescence in our SPOFA procedure assuming a linear relationship between $\mathrm{Ca}^{2+}$ influx and resulting fluorescence (as determined experimentally; see below). In addition, the models containing a single $\mathrm{Ca}^{2+}$ channel were used to compute the distribution of channel open latencies and open times as shown in Figure 4.

\section{Results}

\section{Spatial resolution of data acquisition}

Adult frog NMJs are characterized by long linear nerve terminals containing numerous active zones and thus allow simultaneous imaging of multiple clearly defined active zones subsequent to nerve stimulation (Wachman et al., 2004). Each active zone is $\sim 1$ $\mu \mathrm{m}$ long, is oriented perpendicular to the long axis of the terminal, overlies a postsynaptic junctional fold containing densely packed nicotinic acetylcholine receptors, and is separated from adjacent active zones by $\sim 1 \mu \mathrm{m}$ (shown schematically in Fig. $1 A, B$, left). Approximately 200-250 intramembraneous particles (denoted active zone proteins in Fig. $1 \mathrm{~B}$, left) include $\mathrm{N}$-type voltage-gated $\mathrm{Ca}^{2+}$ channels, are arrayed in parallel double rows running the length of the active zone, and are bordered by $25-40$ docked synaptic vesicles. For $\mathrm{Ca}^{2+}$ imaging (Fig. $1 \mathrm{~B}$, right), terminals were loaded with Calcium Green-1 Dextran (3000 molecular weight, $K_{\mathrm{D}} \sim 540 \mathrm{nM}$ ), and postsynaptic acetylcholine receptors were labeled with Alexa Fluor 594-conjugated $\alpha$-bungarotoxin. Regions of terminals containing well-focused active zones were identified by receptor fluorescence before initiation of low-frequency nerve stimulation $(0.5 \mathrm{~Hz})$ and acquisition of $\mathrm{Ca}^{2+}$ fluorescence.

To determine the effective optical resolution of our imaging system, we measured its PSF using sparsely distributed $20 \mathrm{~nm}$ fluorescent beads. Figure $1 C$ shows that the results were well fit by a Gaussian function and that the full width at half-maximum amplitude was $450 \mathrm{~nm}$. This distance, as indicated schematically in Figure $1 B$ (left), is approximately two to three times larger than the pixel size of our cameras (see Materials and Methods) and approximately half the length of an active zone. Thus, we concluded that each pixel selected for analysis (see below) actually sampled an area approximately equivalent to one-half of an active zone. To confirm this conclusion with $\mathrm{Ca}^{2+}$-dependent fluorescence imaging from a dye-loaded frog nerve terminal, we performed cross-correlation analysis of stimulus-dependent signals detected by adjacent pixels within active zones. As shown in Figure $1 D$, a Lorentzian fit of our results gave a half-width of 410 $\mathrm{nm}$, very close to the value obtained for the PSF. We therefore reasoned that our high-speed fluorescence acquisition system (1.5 ms delay after stimulation, $1 \mathrm{~ms}$ acquisition window; see Materials and Methods) captured signals primarily limited by the optical PSF and not by spatiotemporal spread of the $\mathrm{Ca}^{2+}$ fluorescence. Together, our PSF and cross-correlation results provide strong evidence that subsequent findings from variance analysis correspond to a space approximately equivalent to one-half of an active zone.

\section{Selection of active zone pixels}

We have shown previously that action potential-evoked $\mathrm{Ca}^{2+}$ signals in this preparation are detected in active zones of nerve terminals and arise exclusively from $\mathrm{Ca}^{2+}$ entry through $\mathrm{N}$-type $\mathrm{Ca}^{2+}$ channels, with no contribution from $\mathrm{Ca}^{2+}$-induced $\mathrm{Ca}^{2+}$ release (Wachman et al., 2004). During low-frequency stimulation, large trial-to-trial variability in $\mathrm{Ca}^{2+}$-dependent fluorescence signals was evident in different active zone regions of the nerve terminal. Figure $1 E$ shows $\alpha$-bungarotoxin labeling of postjunctional membrane underlying four to five active zones, and Figure $1 \mathrm{~F}$ shows variability in $\mathrm{Ca}^{2+}$-dependent fluorescence images captured for the same field of view after six different stimuli selected at random from a train of $\sim 100$ (see Materials and Methods).

Highly variable fluorescence signals can result from detection of a small number $(n)$ of $\mathrm{Ca}^{2+}$ channels that function independently and have a small probability $(p)$ of opening during an action potential. In principle, binomial fluctuation analysis can provide estimates of $n$ and $p$ under such conditions, using two independent experimental measurements from which to calculate the two unknowns. For example, early binomial analysis of quantal neurotransmitter release (Del Castillo and Katz, 1954) was based on determination of the failure rate together with the frequencies of single (quantal) and multiple responses. In the present case, however, failures and quantal responses cannot be determined with certainty because the signal-to-noise ratio is very low, i.e., the difference between stimulus-dependent $\mathrm{Ca}^{2+}$ fluorescence and resting background fluorescence is very small. Therefore, a different approach must be taken and we followed previous reports of optical fluctuation analysis (Sabatini and Svoboda, 2000), using two ratios of values (fluorescence intensity and the squared coefficient of variation; see below) measured before and after an experimental manipulation that specifically increases $p$. In this way, one then can calculate $p$ and $n$ without direct determination of the failure rate or the size of a quantal response (see Materials and Methods).

Over the course of repeated trials, the stimulus-dependent mean fluorescence $\left(\bar{f}_{s}\right)$ (Fig. $2 C$ ) and the variability of fluorescence (e.g., $\sigma_{s}$ ) are expected to exceed the corresponding background levels $\left[\bar{f}_{b}\right.$ (Fig. $2 \mathrm{~B}$ ) and $\sigma_{b}$, respectively] for pixels that periodically detect $\mathrm{Ca}^{2+}$ influx through one or more open $\mathrm{Ca}^{2+}$ channels. To apply fluctuation analysis to data obtained from individual pixels (SPOFA), we first had to identify those pixels 

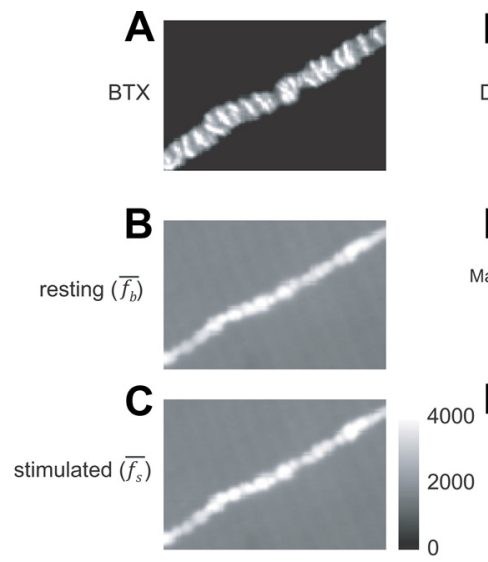
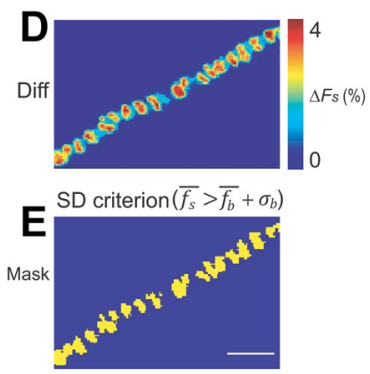

$\mathbf{F}$

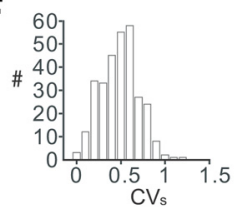

Figure 2. Method used to define active zone regions of the nerve terminal within which action potential-evoked $\mathrm{Ca}^{2+}$ influx is restricted. $\boldsymbol{A}$, Acetylcholine receptor distribution determined using Alexa Fluor 594-conjugated $\alpha$-bungarotoxin. $\boldsymbol{B}$, Resting fluorescence image of a frog motor nerve terminal averaged over 100 trials. $C$, Fluorescence image of the nerve terminal after a single AP stimulation averaged over 100 trials. D, Average (100 trials) $\mathrm{Ca}^{2+}$ influx within active zone regions of the nerve terminal evoked by a single AP. The pattern of $\mathrm{Ca}^{2+}$ influx averaged over 100 stimulus trials decorates nerve terminals with fluorescence signals that can visually identify active zone regions. $E$, Masking the active zone regions with the criterion that pixels have a mean stimulated fluorescence intensity exceeding 1 SD above resting fluorescence (SD criterion). Scale bar, $5 \mu \mathrm{m}$. F, Histogram distribution of CVs over 100 stimulus trials for all pixels selected by the criterion shown in $\boldsymbol{E}$.

that responded to repeated stimulation with both a statistically significant percentage increase in mean fluorescence $\left(\Delta F_{s}=\left(\bar{f}_{s}-\right.\right.$ $\left.\left.\bar{f}_{b}\right) / \bar{f}_{b} \times 100\right)($ Fig. $2 D)$ and also an increase in the variance of fluorescence intensity $\left(\sigma_{s}^{2}-\sigma_{b}^{2}>0\right)$. As expected, increasingly restrictive criteria for statistical significance reduced the number of pixels selected for analysis. In addition, and again as one would expect, the remaining pixels were increasingly restricted to regions directly over active zones (Figs. $1 B$, right, $2 A, E$ ). In fact, we could predict how the results of fluctuation analysis would be influenced by the choice of criterion for statistical significance, particularly with respect to the apparent number $(n)$ of functional $\mathrm{Ca}^{2+}$ channels "seen" by selected pixels. To test our prediction and confirm interpretation of our results, we repeated our analysis using a wide range of significance levels and characterized the impact on calculated values of $n$ and $p$ (see subsequent discussion of Table 1 and Fig. 6 below).

To illustrate one highly selective criterion, Figures $1 B$ (right) and 2 show pixel selection based on a 1 SD method, i.e., for a given pixel to be included in additional analysis, the mean fluorescence after stimulation had to be at least $1 \mathrm{SD}$ above the average background fluorescence (SD criterion, $\bar{f}_{s}>\bar{f}_{b}+\sigma_{b}$ ). As shown, pixels selected by the SD criterion overlie active zone locations identified by postsynaptic acetylcholine receptor staining. As introduced above, SPOFA also requires the use of coefficient of variation values $\left(\mathrm{CV}_{s}=\frac{\sqrt{\sigma_{s}^{2}-\sigma_{b}^{2}}}{\bar{f}_{s}-\bar{f}_{b}}\right)$, and Figure $2 F$ shows the distribution obtained from a representative nerve terminal using pixels that met the SD criterion. In this example, the median value was 0.45 , and similar CVs values were obtained from a total of seven nerve terminals $(0.44 \pm 0.08$, mean $\pm \mathrm{SD}$; range, 0.32-0.54).

\section{Numerical validation of SPOFA}

We wanted to determine $p$ and $n$ based on observed changes in both mean fluorescence and the variability of fluorescence sub- sequent to an experimental manipulation that specifically increases $p$. As detailed in Materials and Methods, we treated the terminal with DAP, which blocks a fraction of voltage-gated potassium channels and thus increases the size and duration of the action potential. Our calculation of $n$ and $p$ from fluorescence measurements is based on the assumption that fluctuations in fluorescence are attributable to a binomial distribution of $n$ channels that open with probability $p$. However, in reality, other sources of variability contribute to the stimulus-dependent fluorescence signal. For example, the amount of $\mathrm{Ca}^{2+}$ flux through an open channel varies because of the distribution of times to channel opening (opening latencies) as well as the distribution of open durations. Through both of these effects, the size and timing of single-channel $\mathrm{Ca}^{2+}$ signals (total, or integrated, single-channel currents) may be affected. Furthermore, because the duration of individual $\mathrm{Ca}^{2+}$ signals may exceed the image acquisition window and may do so by differing amounts, the fraction of total signal captured before and after DAP treatment may also change. Thus, to estimate $p$ and $n$ accurately and establish error estimates for their values, these additional contributions may need to be taken into account.

To assess the possible impact of all these factors on our ability to calculate $p$ and $n$ via binomial analysis, we used computer simulations to investigate the $\mathrm{Ca}^{2+}$ flux through individual channels in response to action potentials (NEURON and MCell simulations; see Materials and Methods). We could then use SPOFA to calculate the values of $p$ and $n$ based on the simulated fluorescence, compare directly with the known values of $p$ and $n$ chosen for the simulation, and then determine error estimates to be used with binomial analysis of our experimental fluorescence measurements.

Figure $3 A$ shows two representative action potentials (AP1 and AP2) under control conditions and in the presence of DAP generated via the NEURON simulation environment (Carnevale and Hines, 2006). As shown in Figure 3B, the generated action potentials agree well with experimental recordings from cultured Xenopus nerve-muscle synapses (Pattillo et al., 2001). We used these action potentials in MCell simulations (Stiles et al., 1996; Stiles and Bartol 2001; Kerr et al., 2008) to drive stochastic voltage-dependent changes in $\mathrm{Ca}^{2+}$ channel conformation, as well as stochastic voltage-dependent $\mathrm{Ca}^{2+}$ ion flux through the channel while it was in the open state. Figure $3 C$ shows corresponding closed-to-open and open-to-closed transitions obtained over the course of repeated trials (cases in which the channel did not open at all are not shown). In the same figure, instances of $\mathrm{Ca}^{2+}$ ion flux through the open state (single, or rarely, multiple ions) during discrete time intervals are also indicated, and it is evident that such permeation events were more likely when the driving force for $\mathrm{Ca}^{2+}$ entry was high. In Figure $3 B$, we compare computed whole-cell $\mathrm{Ca}^{2+}$ currents with recordings from cultured Xenopus nerve-muscle synapses (Pattillo et al., 2001). Both simulated action potential waveforms give rise to currents that match the time course of experimental data well, particularly AP2.

To estimate the amount of $\mathrm{Ca}^{2+}$ signal that would be detected experimentally as mean fluorescence, we chose a $1 \mathrm{~ms}$ time window corresponding to our experimental image acquisition time and then summed all $\mathrm{Ca}^{2+}$ ions that passed through channels before and during the acquisition window. Because experimentally we do not know precisely where within the duration of the action potential our $1 \mathrm{~ms}$ acquisition window falls, we used two different modeling scenarios to bracket the behavior of the system. In the first, the acquisition window starts shortly after action 
A
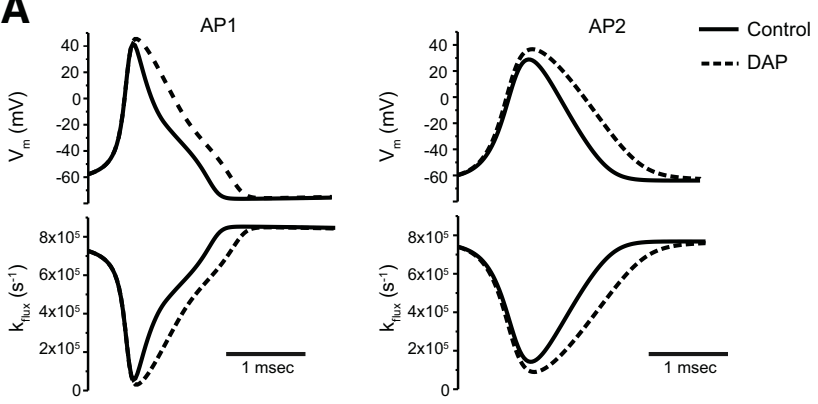

B

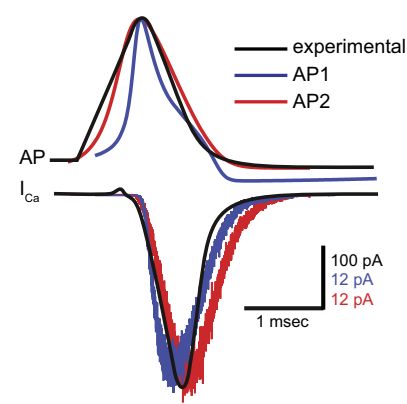

C $\quad \mathrm{C}_{1} \leftrightarrow \mathrm{C}_{2} \leftrightarrow \mathrm{C}_{3} \leftrightarrow \mathrm{O}$

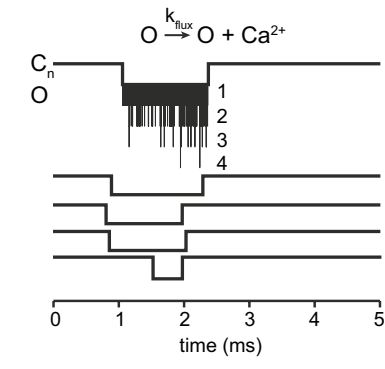

Figure 3. Simulated action potentials and computational model of action-potentialtriggered $\mathrm{Ca}^{2+}$ influx. $\boldsymbol{A}$, Simulation program NEURON generated action potentials AP1 and AP2 under control conditions and in the presence of DAP. The bottom panels show the $\mathrm{Ca}^{2+}$ flux $k_{\text {flux }}$ through the open $\mathrm{Ca}^{2+}$ channels proportional to the driving force $V_{\mathrm{m}}-E_{\mathrm{Ca}} \cdot \boldsymbol{B}$, Comparison of AP1 (blue) and AP2 (red) with an experimentally recorded action potential from cultured Xenopus nerve-muscle synapses (black) (Pattillo et al., 2001) and the corresponding whole-cell $\mathrm{Ca}^{2+}$ currents simulated (blue and red) and recorded from a presynaptic cultured neuromuscular junction varicosity (black) (Pattillo et al., 2001). C, The top depicts a schematic diagram of our kinetic $\mathrm{Ca}^{2+}$ channel model with three closed and one open state. The open state conducts $\mathrm{Ca}^{2+}$ ions according to the driving force given by $k_{\text {flux }}$ (see also $A$ ). Below, we show plots of the channel open duration for five representative simulations. In addition, the first plot gives the number of $\mathrm{Ca}^{2+}$ ions conducted per unit time interval.

potential onset after the integrated $\mathrm{Ca}^{2+}$ current has reached 5\% of its maximum value. We call this early acquisition. In the second, acquisition starts only after the integrated $\mathrm{Ca}^{2+}$ current has reached $50 \%$ of its maximum value. Here, our $1 \mathrm{~ms}$ acquisition window captures most of the total $\mathrm{Ca}^{2+}$ signal entering the terminal during the action potential. We call this second scenario late acquisition.

Initially, we simulated models containing a single $\mathrm{Ca}^{2+}$ channel. To illustrate the data obtained over repeated trials, Figure 4 shows histograms of channel opening latencies (Fig. $4 \mathrm{~A}$ ), channel open times (Fig. $4 B$ ), and total single-channel $\mathrm{Ca}^{2+}$ ion passage (current integral, Fig. $4 \mathrm{C}$ obtained via late acquisition) obtained during repeated trials for the two action potential waveforms AP1 and AP2. The mean channel open times under control conditions (AP1, $0.56 \pm 0.3 \mathrm{~ms} ; \mathrm{AP} 2,0.65 \pm 0.35 \mathrm{~ms}$ ) and in the presence of DAP (AP1, $0.77 \pm 0.34$; AP2, $0.91 \pm 0.4$ ) are below $1 \mathrm{~ms}$, indicating that our $1 \mathrm{~ms}$ acquisition window is sufficient to capture most of the $\mathrm{Ca}^{2+}$ signal particularly in the late acquisition scenario (however, see discussion of current distribution below). Figure $4 C$ depicts the $\mathrm{Ca}^{2+}$ current distributions generated with AP1 and AP2 under control and DAP conditions. Overall, the current distributions under both conditions are similar. The fact that, in the presence of DAP, we sample slightly lower maximum current values is attributable to the prolonged action potential duration and correspondingly lower driving force combined with a fixed $1 \mathrm{~ms}$ acquisition window.
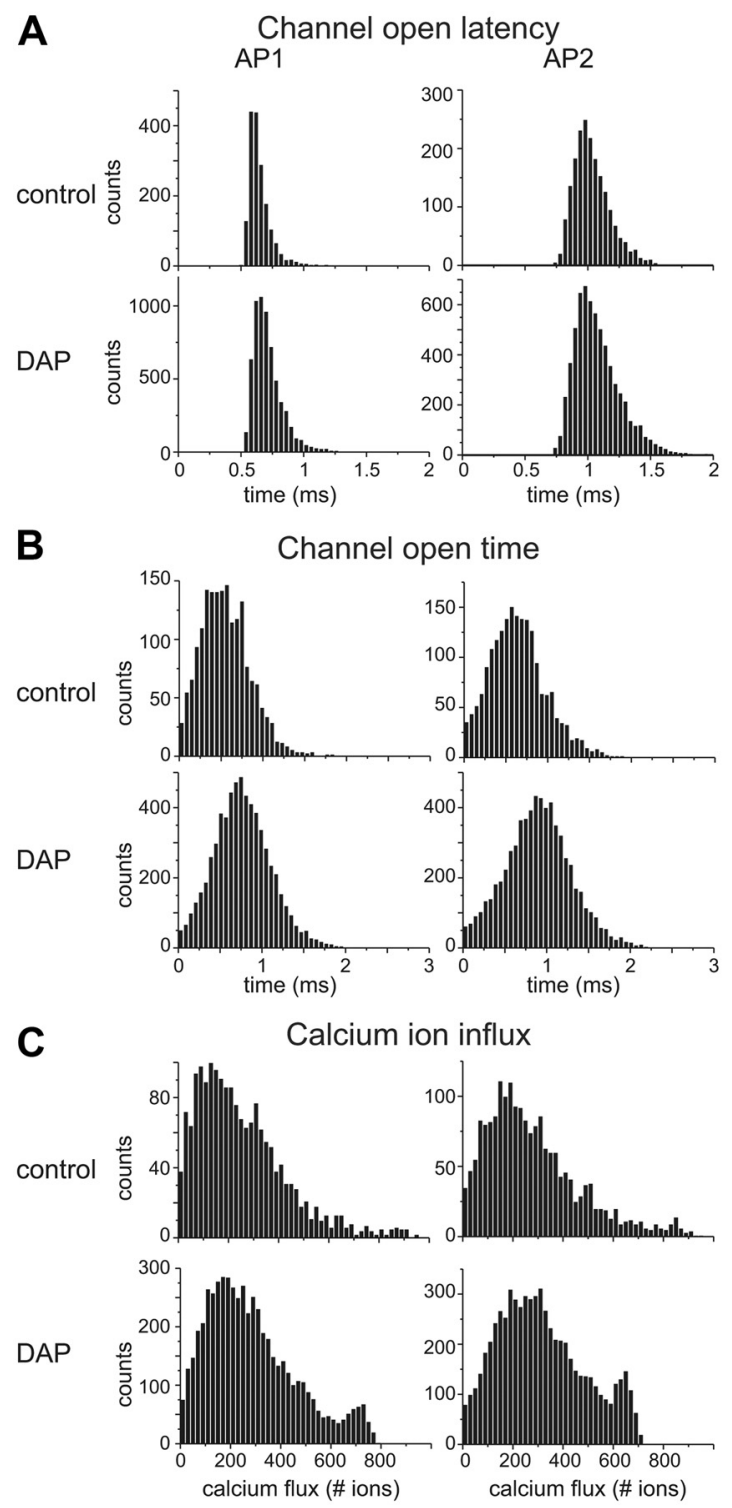

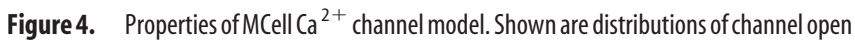
latencies $(\boldsymbol{A})$, channel open times $(\boldsymbol{B})$, and $\mathrm{Ca}^{2+}$ current integrals $(\boldsymbol{C})$ for AP1 and AP2 under both control and DAP conditions using the late acquisition scenario (see Results). The "dimples" observed in the DAP plots for Care an artifact of our $1 \mathrm{~ms}$ acquisition window and the decrease in driving force for DAP-modified APs that depolarize to potentials closer to the modeled calcium channel equilibrium potential, followed by a slightly later return to a strong driving force as the AP repolarizes.

Our next step in modeling studies was to use multiple $\mathrm{Ca}^{2+}$ channels in each simulation and to determine the time course of the summed $\mathrm{Ca}^{2+}$ signal for different combinations of action potential waveform, number of channels, and with or without DAP treatment. For each set of conditions, we ran thousands of simulations and obtained the average number of permeant $\mathrm{Ca}^{2+}$ ions as a function of time (data not shown). The resulting $\mathrm{Ca}^{2+}$ currents were then used to compute values for $p$ and $n$ according to the SPOFA procedure outlined in Materials and Methods. We used the number of $\mathrm{Ca}^{2+}$ ions summed over a $1 \mathrm{~ms}$ acquisition window as proxy for measured fluorescence. As an example, Table 2 gives values for summed $\mathrm{Ca}^{2+}$ ions, $\mathrm{CV}$ values, $R_{F}, R_{\mathrm{CV}}$, and calculated values of $p$ for a system driven by action potential AP1 under an early acquisition scenario for simulation systems containing $1,10,15,20,30$, or $40 \mathrm{Ca}^{2+}$ channels. The computed 
Table 2. Statistical properties of computational calcium channel model

\begin{tabular}{lccllllll}
\hline$n$ & $\begin{array}{l}\mathrm{C}^{2+} \text { count } \\
\text { control }\end{array}$ & $\begin{array}{l}\mathrm{Ca}^{2+} \text { count } \\
\text { DAP }\end{array}$ & $R_{F}$ & $\mathrm{CV}^{2}$ & $\mathrm{CV}_{\text {DAP }}^{2}$ & $R_{\mathrm{CV}^{2}}$ & $p$ & $n$ \\
\hline 1 & 44 & 144 & 0.306 & 6.96 & 1.25 & 5.57 & 0.15 & 0.74 \\
10 & 443 & 1407 & 0.315 & 0.72 & 0.13 & 5.56 & 0.17 & 7.18 \\
15 & 660 & 2122 & 0.311 & 0.49 & 0.09 & 5.69 & 0.16 & 10.59 \\
20 & 889 & 2821 & 0.315 & 0.35 & 0.06 & 5.58 & 0.17 & 14.56 \\
30 & 1339 & 4248 & 0.315 & 0.24 & 0.04 & 5.47 & 0.16 & 21.88 \\
40 & 1770 & 5676 & 0.312 & 0.18 & 0.03 & 5.74 & 0.17 & 28.37 \\
Avg. & & & & & & & 0.16 & \\
\hline
\end{tabular}

Shown are data from a model driven by a NEURON-generated AP (AP1) (Fig. 3) and our stochastically gated channel model shown in Figure 3. The actual open probabilities in the model are $p=0.18$ and $p_{D A P}=0.63$ for control and after DAP treatment, respectively. The data were acquired with a $1 \mathrm{~ms}$ sampling window, and data collection started after the current integral reached $5 \%$ of its maximum value (early acquisition; see Results).

Table 3. Average relative errors for calculated values of $p$ and $n$ with respect to the known values

\begin{tabular}{lcc}
\hline Simulation type & $\begin{array}{l}\text { Average relative } \\
\text { error in } p\end{array}$ & $\begin{array}{l}\text { Average relative } \\
\text { error in } n\end{array}$ \\
\hline AP1 (early acquisition) & -0.091 & -0.278 \\
AP1 (late acquisition) & -0.343 & -0.019 \\
AP2 (early acquisition) & 0.032 & -0.326 \\
AP2 (late acquisition) & -0.351 & 0.068 \\
\hline
\end{tabular}

Each row corresponds to a simulation using one of the two action potential waveforms. Positive and negative values for relative errors signify over and underestimates obtained via optical fluctuation analysis, respectively.

value of $p$ across all these systems was fairly consistent with a mean of $p$ of $\sim 0.16$, in good agreement with the actual value of $p=0.18$ used in the simulations. The last column in Table 2 gives the corresponding values for $n$ computed via Equation 11 using the computed average $p$ value. Thus, in this particular scenario, SPOFA underestimates the true values of $n$ by $\sim 25 \%$.

Finally, in Table 3, we summarize the relative errors in the computed values of $p$ and $n$ for models driven by action potential waveforms AP1 and AP2 under both early and late acquisition scenarios. Based on these results, we can infer that the early acquisition scenario provides good estimates of $p$ but tends to underestimate $n$ by up to $30 \%$. Conversely, the late acquisition scenario tends to underestimate the value of $p$ by up to $30 \%$ but results in good estimates of $n$. Because our experimental acquisition window lies somewhere between these two extremes, we can thus argue that SPOFA applied to our experimental data provides reliable estimates of both $p$ and $n$ and will at most underestimate either one by $\sim 30 \%$. These findings are consistent for both action potential waveforms studied, suggesting that the predictive power of our SPOFA method is independent of the detailed shape of the underlying action potential when it is within these ranges.

\section{The probability $(p)$ that $\mathrm{Ca}^{2+}$ channels open during an action potential}

Our use of SPOFA to determine $p$ and $n$ from single-pixel fluorescence measurements was based on the use of two ratios, for which each component value was measured before and after an experimental manipulation that specifically increased $p$. Preparations were treated with $5 \mu \mathrm{M}$ DAP for 30 min to block potassium channels selectively and, as a result, broaden the action potential (Bostock et al., 1981; Thomsen and Wilson, 1983; Augustine, 1990) and secondarily increase $p$ (Augustine, 1990).

Figure 5, $A$ and $B$, shows $\mathrm{Ca}^{2+}$ imaging data from a representative nerve terminal before and after DAP exposure. Using our $\mathrm{SD}$ criterion to select pixels, we calculated per pixel values of $\Delta F_{s}$ and $\mathrm{CV}_{s}$ under both conditions, and Figure 5, $C$ and $D$, shows the corresponding frequency distributions. Although DAP exposure
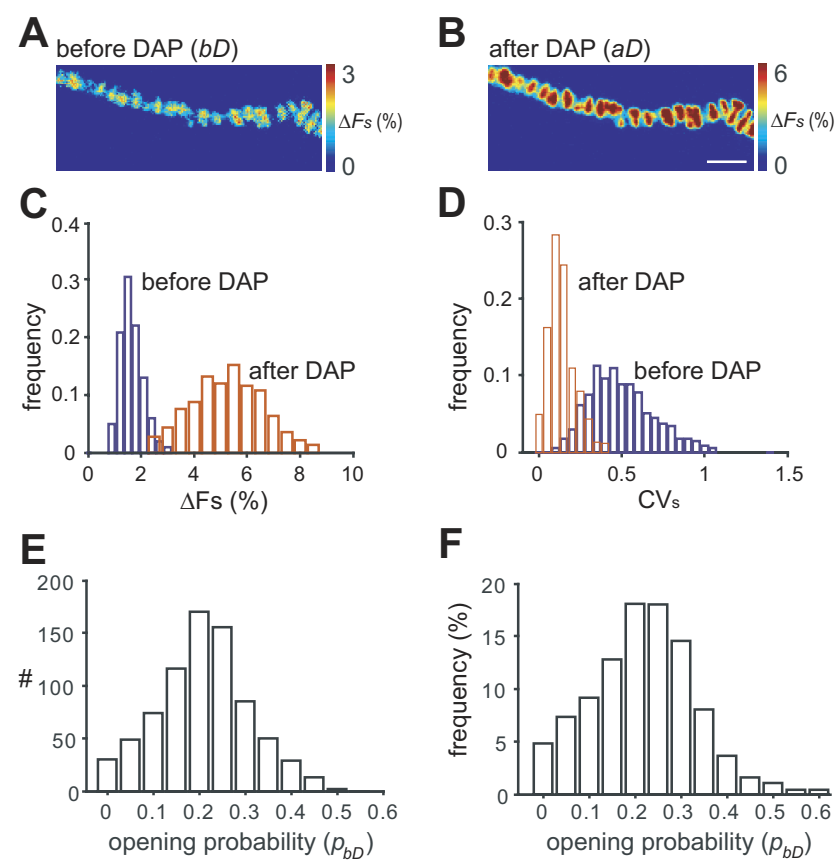

Figure 5. Opening probability of $\mathrm{Ca}^{2+}$ channels during an action potential. $\boldsymbol{A}$, Averaged AP-evoked $\mathrm{Ca}^{2+}$ influx under control condition (90 trials). B, Averaged AP-evoked Ca ${ }^{2+}$ influx after $5 \mu \mathrm{m}$ 3,4-diaminopyridine treatment (90 trials). Scale bar, $5 \mu \mathrm{m}$. C, Histogram distribution of the fluorescence intensity reported by individual pixels before (blue) and after (red) exposure to DAP. D, Histogram distribution of the stimulus-dependent coefficient of variation of individual pixels before (blue) and after (red) DAP exposure. $\boldsymbol{E}$, Histogram distribution plotting opening probability of $\mathrm{Ca}^{2+}$ channels during an action potential calculated from individual pixels for the example control terminal shown in $\boldsymbol{A}$. $\boldsymbol{F}$, Histogram distribution plotting the average opening probability of $\mathrm{Ca}^{2+}$ channels during an action potential calculated from individual pixels pooled from a total of four control nerve terminals.

strongly increased $\Delta F_{D s}$, it simultaneously decreased $\mathrm{CV}_{D s}$ compared with control, and both of these effects are consistent with an increase in $p$ according to standard binomial theory. In brief, if $n \mathrm{Ca}^{2+}$ channels are detected by a pixel and open independently with an average probability $p$ during an action potential, the expected number of open channels per trial will follow a binomial distribution. The mean of a binomial distribution $\mu$ is given simply by $n p$, and so an increase in $p$ will clearly increase $\mu$, or, in our case, the mean value of $\Delta F_{s}$. The binomial variance $\sigma^{2}$ and coefficient of variation $\mathrm{CV}$ are given by $(1-p) n p$ and $\frac{1-p}{n p}$, respectively. From the latter, an increase in $p$ will decrease $\mathrm{CV}$, or $\mathrm{CV}_{s}$ in our experiments.

To estimate $p$, we used the ratio of $\Delta F_{s}$ and $\Delta F_{D s}$, together with the ratio of $\mathrm{CV}_{s}^{2}$ and $\mathrm{CV}_{D s}^{2}$, measured before and after DAP treatment. These calculations allow us to obtain values for $p$ both before (control) and after the DAP treatment (see Eqs. 14 and 15 in Materials and Methods). Figure $5 E$ shows the distribution of control values obtained from the data shown previously for a representative nerve terminal in Figure $5 A-D$. Because the distribution was not Gaussian, we used the median value (0.22) as our estimate of the mean opening probability $(p)$ for $\mathrm{Ca}^{2+}$ channels in this nerve terminal. After DAP treatment, the estimated mean opening probability increased to 0.72 . Similar results were obtained from four nerve terminals for which we pooled all the single-pixel data and generated a frequency histogram for $p$ under control conditions (Fig. $5 F$ ). The median value was 0.24 for the pooled data. Taking into account our numerically derived error estimates (see above, Numerical validation of SPOFA), 
we can derive a lower limit of 0.23 and an upper limit of 0.37 for $p$ with the actual value likely to be somewhere between.

\section{The number $(n)$ of $\mathrm{Ca}^{2+}$ channels in active zones}

We based our initial estimates of $n$ on pixel data obtained from the four nerve terminals used in the DAP studies outlined above. For each terminal, the median $p$ and $\mathrm{CV}_{s}^{2}$ values were used to calculate a value of $n$ (see Eq. 16 in Materials and Methods; pixel selection was performed using the SD criterion). Using this approach, we found that $n$ ranged from 14 to 24 across the four terminals, with a mean \pm SD value of $18.3 \pm 4.8$. When values of $n$ were calculated individually for each pixel and then averaged together, the results were not significantly different.

In addition, we analyzed the seven nerve terminals not treated with DAP discussed previously (see above, Selection of active zone pixels). Because these terminals were not exposed to DAP, we did not have an independent estimate of $p$. Instead, we used the median value $(p=$ 0.24 ) determined from the DAP experiments on the initial four terminals. Using this value for $p$, together with the median $\mathrm{CV}_{s}^{2}$ value for each untreated terminal, we calculated values of $n$, which ranged from 11 to 31 , with a mean \pm SD value of $18.0 \pm 7.5$. This average was not significantly different from the result obtained with the DAP-treated terminals (unpaired Student's $t$ test, $p=0.9$ ). Thus, we pooled the data from all 11 nerve terminals to obtain a final estimate of $n, 18.1 \pm 6.4$ (mean $\pm \mathrm{SD}$ ). From our previous characterization of the PSF and pixel correlation analysis (Fig. 1), we estimated that single pixels sample spatial regions approximately equivalent to one-half of an active zone. Thus, our data indicate that each active zone has $\sim 2 n$, or only $\sim 36 \mathrm{Ca}^{2+}$ channels that are capable of opening in response to an action potential (functional channels). Taking into account our numerically derived error estimates (see above, Numerical validation of SPOFA), we can compute a lower limit of 34 and an upper limit of 53 for the number of $\mathrm{Ca}^{2+}$ channels, with the actual value likely falling somewhere between. Presumably, these functional channels are distributed among the 200-250 intramembraneous particles described in freeze-fracture replicas.

\section{The impact of pixel selection on estimates of $p$ and $n$}

Given that we used up to 100 nerve stimuli during image acquisition and also that individual pixels sampled an area of approximately half an active zone, we had two primary expectations regarding the selection of pixels for analysis. First, notwithstanding the low signal-to-noise ratio, a relatively low level of statistical signif-

A

$\mathrm{P}<10^{-9}$ $p<1)$.

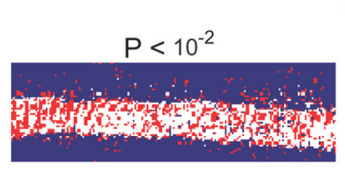

B
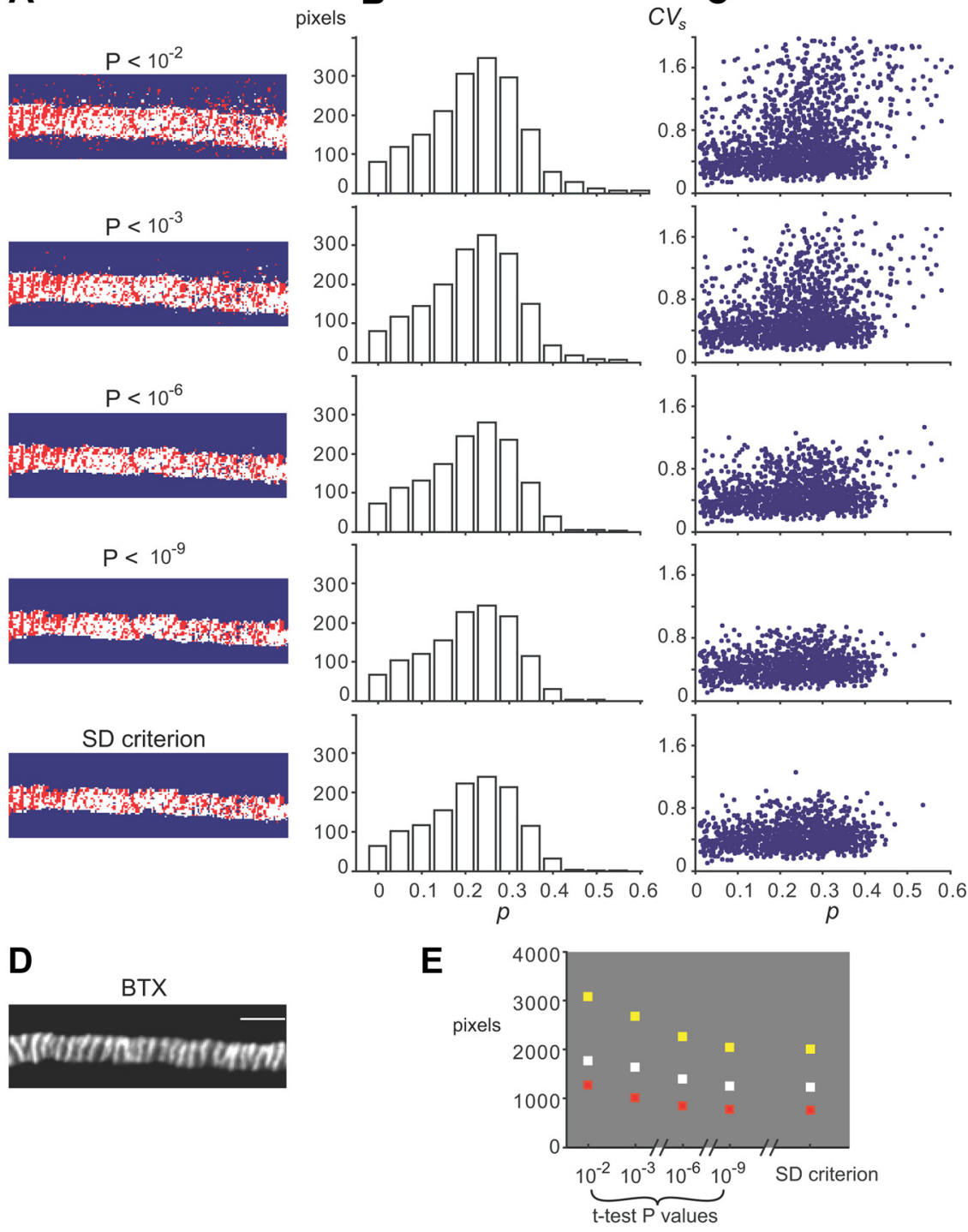

Figure 6. Varying the criterion for pixel selection does not bias our estimate for the probability that $\mathrm{Ca}^{2+}$ channels open during an action potential. $\boldsymbol{A}$, Mask for the pixels selected after using different criteria, i.e., SD criterion, or a Student's $t$ test using different in white could be used to derive a meaningful $p$ value (i.e., $0<p<1$ ). $\boldsymbol{B}$, Histogram distribution of opening probability calculated from the selected pixels under each criterion. $\boldsymbol{C}$, In each graph, $\mathrm{CV}_{s}$ is plotted against opening probability to reveal subsets of the channels during an $A P$ is not changed as more restrictive criteria eliminate a subset of the data. $\boldsymbol{D}$, Predicted active zone regions of the example nerve terminal identified by labeling the postsynaptic acetylcholine receptor clusters with Alexa Fluor $594-\alpha-$ (yellow) and the corresponding subset of these pixels that either do (white) or do not (red) resolve a meaningful $p$ value (i.e., $0<$

icance should be able to differentiate between pixels that overlie the nerve terminal as opposed to the surrounding tissue. Second, as the criterion for statistical significance became more restrictive and fewer pixels were selected, those remaining should be completely restricted to the nerve terminal, and, in addition, should be increasingly restricted to positions centered over active zones (and therefore over functional $\mathrm{Ca}^{2+}$ channels). We then reasoned that values calculated for $n$ should be smaller with less restrictive selection criteria, because an increasing fraction of pixels would "see" an area on the periphery of an active zone and therefore would detect a fluorescence signal originating from fewer channels. Conversely, values calculated for $p$ should remain fairly con- 
A
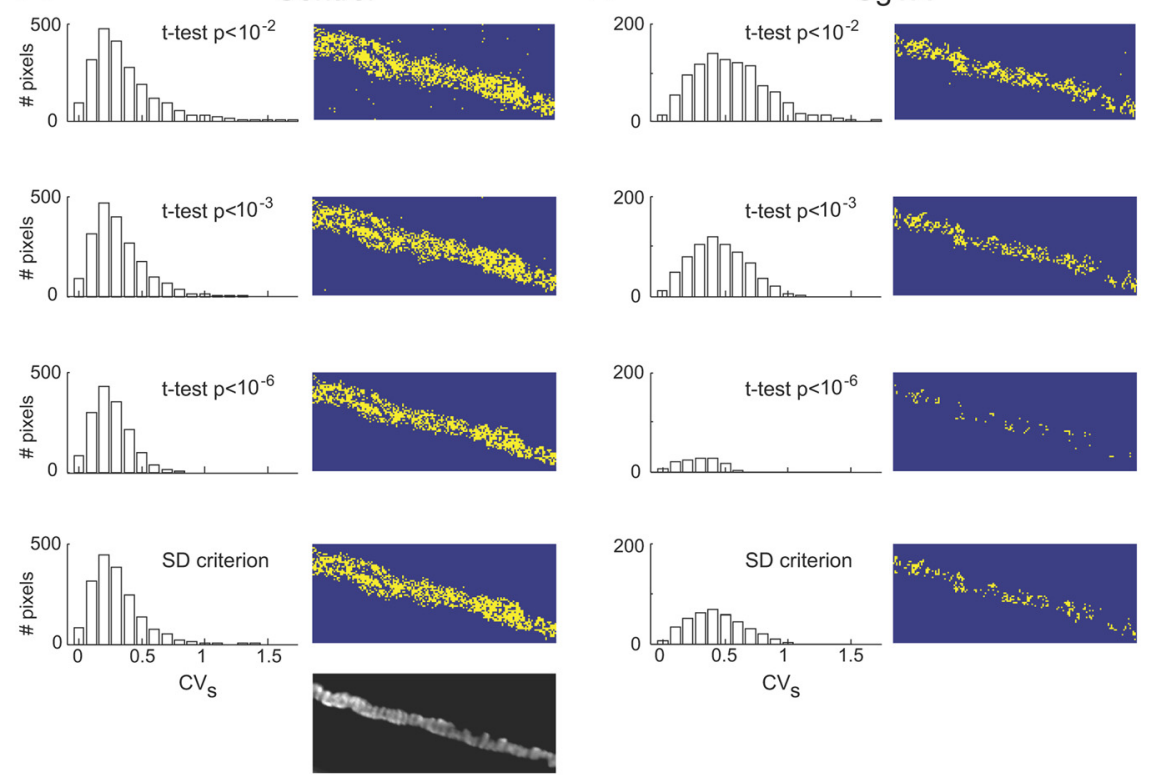

C

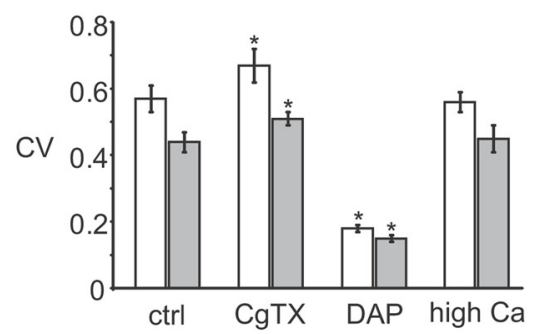

B
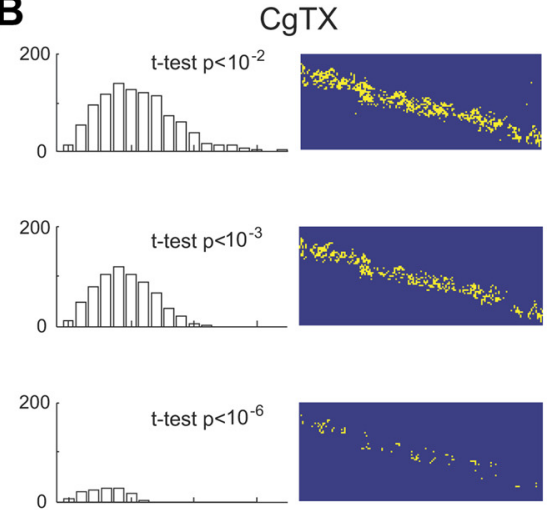

D

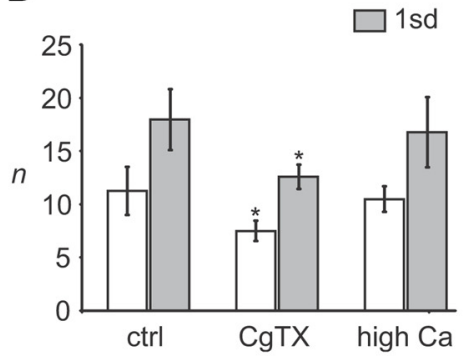

Figure 7. Effects of $\mathrm{CgTX}_{\mathrm{T}}$ on $\mathrm{CV}_{s}$ measured using a variety of pixel selection criteria and the summary data for measured $\mathrm{CV}_{s}$ values and calculated $n$ values under different treatment conditions. A, Distribution of $\mathrm{CV}_{s}$ values (left) and mask for the pixels selected after using different criteria (right), i.e., SD criterion, or a Student's $t$ test using different significance levels $\left(p<10^{-2}\right.$, $10^{-3}$, or $10^{-6}$ ) from a representative nerve terminal under control conditions. The grayscale image at the bottom represents the predicted active zone regions of the nerve terminal identified by labeling the postsynaptic acetylcholine receptor clusters with Alexa Fluor $594-\alpha$-bungarotoxin. $B$, Distribution of $\mathrm{CV}_{s}$ values (left) and mask for the selected pixels from the same nerve terminal as is $A$ after treatment with $100 \mathrm{~nm}$ CgTX for 30 min. C, Summary plot of the calculated $\mathrm{CV}_{s}$ values (mean \pm SEM) for pixels sampling

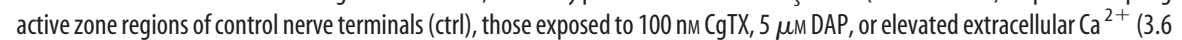
$\mathrm{mm}$; high $\left.\mathrm{Ca}^{2+}\right)$ when using either the SD criterion (gray bars) or a Student's $t$ test $(p<0.01$; white bars). $\boldsymbol{D}$, Summary plot of the calculated number of $\mathrm{Ca}^{2+}$ channels $(n)$ sampled by active zone pixels (mean \pm SEM) under control conditions (ctrl), after

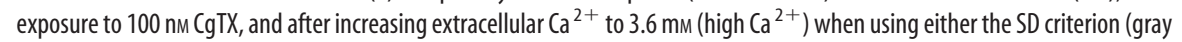
bars) or a Student's t test $\left(p<0.01\right.$; white bars). ${ }^{*} p<0.05$, significantly different from control, one-way ANOVA with Tukey's post hoc test.

stant over a wide range of selection criteria, assuming that functional channels have identical gating characteristics regardless of their location in the active zone, or that any possible heterogeneity of gating function occurs over a spatial scale much smaller than the resolution of our optical system.

Figure 6 and Table 1 summarize the results that verify our predictions for the impact of pixel selection on estimates of $p$ and $n$. Whereas the data shown in Figure 6 are based on a single terminal treated with DAP, Table 1 includes final average values of $p$ and $n$ calculated from multiple DAP-treated terminals (additional analysis of the data presented previously in Fig. 5). Figure $6 A$ (top) shows that a significance criterion as permissive as $p<$ $10^{-2}$ (Student's $t$ test) selected an appreciable number of pixels that lay beyond the borders of the nerve terminal itself (compare position of red and white dots to fluorescence image of postsyn- aptic bungarotoxin stain in Fig. 6D). Values of $\mathrm{CV}_{s}$ and $p$ were widely scattered (Fig. 6B, $C$, top), although clustering was still apparent and indicated a midrange value for $p$ of $\sim 0.2$. When more restrictive criteria were used to determine significance $\left(p<10^{-3}, 10^{-6}\right.$, or $10^{-9}$, or using our SD criterion instead), the number of pixels selected for analysis decreased (Fig. $6 E$ ), the pixels were increasingly restricted to the nerve terminal and active zones (Fig. 6A, top to bottom), and clustering of $\mathrm{CV}_{s}$ and $p$ values became evident (Fig. $6 \mathrm{C}$, top to bottom). The number of selected pixels converged toward an apparent minimum obtained with either $p<10^{-9}$ (Student's $t$ test) or our SD criterion (Fig. $6 E$; recall that the SD criterion was highly restrictive because inclusion of a pixel required that its average fluorescence was greater than the average background value by more than $1 \mathrm{SD}$ ). Figure $6, A$ and $E$, also shows how pixels were subdivided into those that had increased (white) or decreased (red) variance compared with background variance levels ( $\sigma_{s}^{2}$ vs $\sigma_{b}^{2}$ ), despite the prerequisite increase in average fluorescence. Although the presence of pixels with decreased variance at first may seem counterintuitive, in fact it is expected because of the very small absolute size of the stimulus-dependent fluorescence signal $\left(\Delta F_{s}\right.$ of only several percent) (Figs. 1, 2, 5). Unlike a system at the opposite extreme, in which there is a clearly discernible quantal response, here, because of the low open probability $p$ and correspondingly small increase in fluorescence, one expects only a small excess of pixels with increased variance, whereas the remainder may show decreased variance simply by random chance. An examination of the total number of pixels with increased (white squares) and decreased (red squares) variance shown in Figure $6 E$ reveals that this was the case for our data, and careful inspection also shows a minor trend toward a higher fraction of pixels with increased variance as the criterion for selection became more restrictive. Limiting our analysis to selected pixels with increased variance, we found, as predicted, that average values obtained for $p$ were virtually indistinguishable regardless of the pixel selection criterion (Table 1). Table 1 also shows that, as predicted, average values obtained for $n$ increased as the criterion for pixel selection became more restrictive, until nearly identical results were obtained with the two most selective criteria.

\section{Control measurements}

Our SPOFA depends on a linear relationship between $\Delta F_{s}$ and $\Delta\left[\mathrm{Ca}^{2+}\right]$. To confirm the linearity of our measurements, we varied extracellular $\mathrm{Ca}^{2+}$ and measured the resulting change in fluorescence. After doubling extracellular $\mathrm{Ca}^{2+}$ concentration 
(from 1.8 to $3.6 \mathrm{~mm}$, enhancing $\mathrm{Ca}^{2+}$ influx per open channel), the $\mathrm{Ca}^{2+}$ signal detected within active zone regions of the nerve terminal increased by $102 \pm 2 \%(n=4$ terminals $)$. In contrast, the $\mathrm{CV}_{s}$ value obtained with high extracellular $\mathrm{Ca}^{2+}$ was not significantly different from that obtained with normal extracellular $\mathrm{Ca}^{2+}(p>0.05$, one-way ANOVA with Tukey's post hoc test) (Fig. $6 \mathrm{~A}$ ). This doubling in $\mathrm{Ca}^{2+}$ signal, coupled with an unchanged $\mathrm{CV}_{s}$, supports the conclusion that the reduced $\mathrm{CV}_{s}$ obtained after DAP exposure was indeed caused by an increase in $p$ and not by a potential saturation of measured fluorescence. Based on this analysis, we can also conclude that the concentration of extracellular $\mathrm{Ca}^{2+}$ (and thus the magnitude of $\mathrm{Ca}^{2+}$ influx through open channels) does not alter our quantitative analysis of variability in presynaptic fluorescence signals. Therefore, this experiment serves as a control for the validity of our fluctuation analysis.

A lack of significant dye saturation during low-frequency stimulation might be expected given the ordered geometry of adult frog NMJ active zones (long, linear arrays of presynaptic $\mathrm{Ca}^{2+}$ channels distributed spatially at some distance from one another) (Fig. 1), combined with the prediction that there are few $\mathrm{Ca}^{2+}$ channel openings within each active zone during a single action potential (Wachman et al., 2004; this study). The absence of significant dye saturation under similar imaging conditions at this synapse was also reported by Shahrezaei et al. (2006). The linearity of fluorescence and $\mathrm{Ca}^{2+}$ signal in our experiments is fortuitous, and both fluorescence detection and calcium-sensitive dye interactions with calcium ions are expected to saturate outside our experimental conditions.

Finally, to further validate our fluctuation analysis, we also examined the effects of exposure to a submaximal concentration of $\omega$-conotoxin GVIA (CgTX) (100 nM). This treatment is known to completely and irreversibly block (over the course of our experiments) a subset of $\mathrm{N}$-type $\mathrm{Ca}^{2+}$ channels without affecting the probability of opening for channels that remain unblocked (Kerr and Yoshikami, 1984; Stocker et al., 1997). Exposure to 100 nM CgTX blocked total action-potential-evoked $\mathrm{Ca}^{2+}$ entry in the nerve terminal by $70 \pm 5.9 \%$ (mean \pm SD, 5 nerve terminals). As a result, the stimulus-dependent fluorescence signals were very weak, and we had to use a less restrictive pixel selection criterion to be able to select a sufficient number of pixels for analysis. Using a Student's $t$ test to select active zone pixels $(p<0.01)$ (Fig. 7, Table 4), the average $\mathrm{CV}_{s}$ after CgTX treatment increased significantly above control to a value of $0.67 \pm 0.11$ (one-way ANOVA with Tukey's post hoc test, $p<0.05$; mean \pm $\mathrm{SD}, 5$ nerve terminals). Using the estimated $p(0.24)$ determined above (CgTX does not affect the gating properties of unblocked channels) and the $\mathrm{CV}_{s}$ from CgTX-treated nerve terminals, the average $n(n=7.5 \pm 2.1)$ was significantly decreased compared with control (one-way ANOVA with Tukey's post hoc test, $p<$ $0.05)$. The difference in magnitude of CgTX-mediated reduction of total $\mathrm{Ca}^{2+}$ indicator fluorescence $(\sim 70 \%)$ and the reduction in calculated $\mathrm{Ca}^{2+}$ channel number $(\sim 33 \%)$ is attributable to the following. When measuring total $\mathrm{Ca}^{2+}$ indicator fluorescence, we observe the change in the total stimulus-dependent fluorescence within the entire nerve terminal area. This includes some active zone regions of the nerve terminal in which all $\mathrm{Ca}^{2+}$ chan-
Table 4. Pixel selection criteria and their impact on our estimates of $\mathrm{CV}_{s}$ and $n$ in CgTX-treated nerve terminals

\begin{tabular}{llr}
\hline Pixel selection criteria & $\mathrm{CV}_{s}$ & \multicolumn{1}{c}{$n$} \\
\hline Student's $t$ test, $p<10^{-2}$ & $0.67 \pm 0.11$ & $7.5 \pm 2.1$ \\
Student's $t$ test, $p<10^{-3}$ & $0.58 \pm 0.09$ & $10.0 \pm 2.8$ \\
Student's $t$ test, $p<10^{-6}$ & $0.48 \pm 0.11$ & $15.9 \pm 8.1$ \\
SD criterion & $0.51 \pm 0.05$ & $12.6 \pm 2.6$ \\
\hline
\end{tabular}

Figure $7 B$ displays the impact of these selection criteria on the number of pixels selected for analysis in a representative nerve terminal (values represent mean $\pm S D ; n=5$ terminals).

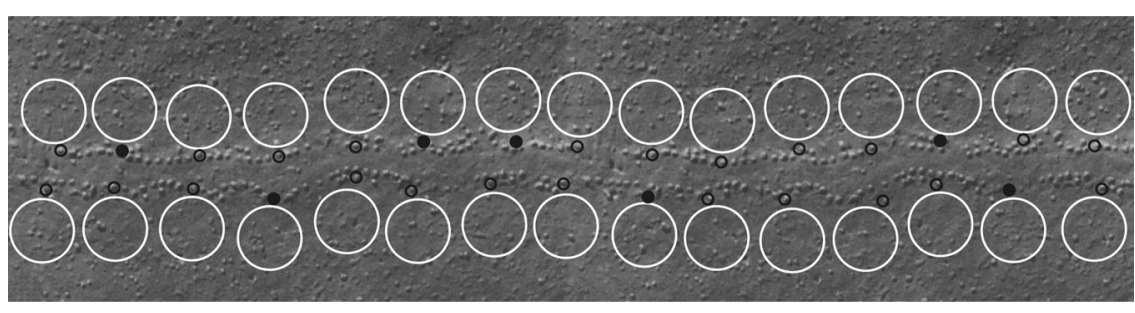

Figure 8. Conceptual model of a single active zone showing graphically the stoichiometric and functional relationship between representation of the hypothesized number and position of associated synaptic vesicles (white circles), voltage-gated $\mathrm{Ca}^{2+}$ (black circles), and the number of these voltage-gated $\mathrm{Ca}^{2+}$ channels that are predicted to open after stimulation by a

nels were completely blocked by the CgTX treatment and others in which some $\mathrm{Ca}^{2+}$ channels remained unblocked after exposure to CgTX. In contrast, our SPOFA analysis can only consider pixels in which some signal remains after CgTX treatment. Under these conditions, the SPOFA analysis underestimates the reduction in $n$ that occurs because there are some pixels that sample active zone regions of the nerve terminal in which $n$ drops to zero after CgTX treatment, and these are not included in the SPOFA analysis. Regardless of this quantitative difference, a decrease in $n$ is consistent with the expected effects of CgTX. Therefore, our method of variance analysis is sensitive to treatments known to alter either $p$ (DAP) or $n$ (CgTX), because the measured $\mathrm{CV}_{s}$ values vary as expected (Fig. 7).

\section{Discussion}

In this study, we have combined variance analysis with highresolution $\mathrm{Ca}^{2+}$ imaging techniques and Monte Carlo simulations to estimate the number and probability of opening of $\mathrm{Ca}^{2+}$ channels within presynaptic active zones. Our results predict that a single active zone at the frog NMJ contains a relatively small number of $\mathrm{Ca}^{2+}$ channels, each with low probability of opening during an action potential.

\section{The opening probability of active zone $\mathrm{Ca}^{2+}$ channels during action potential stimulation}

The probability that presynaptic $\mathrm{Ca}^{2+}$ channels open in response to a single action potential is primarily determined by the activation kinetics of $\mathrm{Ca}^{2+}$ channels and the shape of the action potential. This is distinct from the probability that a channel opens in response to a prolonged voltage step $\left(P_{\mathrm{o}}\right)$, which typically is calculated as the steady-state fraction of time that the channel is in the open state. Here we are interested in the likelihood that a $\mathrm{Ca}^{2+}$ channel will open during a brief action potential depolarization. Indeed, by broadening the action potential with DAP, we have shown that the opening probability of presynaptic $\mathrm{Ca}^{2+}$ channels increase significantly. In a limited number of cases, it has been possible to estimate the opening probability of presyn- 
aptic $\mathrm{Ca}^{2+}$ channels from patch-clamp recordings of $\mathrm{Ca}^{2+}$ current. At the squid giant synapse (Augustine, 1990), the chick ciliary ganglion calyx (Bertram et al., 1996), and cultured Xenopus neuromuscular varicosities (Poage and Meriney, 2002), it has been estimated that opening probability is relatively small (0.10.3 ). In contrast, evidence from the rat calyx of Held (Borst and Sakmann, 1998), hippocampal mossy fiber boutons (Bischofberger et al., 2002), and cerebellar granule cell synapses onto Purkinje neurons (Sabatini and Regehr, 1997) indicate that action potentials activate a large fraction of available $\mathrm{Ca}^{2+}$ current $(0.7-0.9)$. Thus, it is likely that the proportion of presynaptic $\mathrm{Ca}^{2+}$ channels that open with action potential stimulation may be variable between synaptic preparations. Furthermore, $\mathrm{Ca}^{2+}$ channels have characteristic gating kinetics that depend on $\mathrm{Ca}^{2+}$ channel type, splice variant, and auxiliary subunits (Jones and Marks, 1989; Lin et al., 1997, 1999). In fact, striking differences in the rate of activation ( $\sim 15$-fold) have been observed when comparing isoforms of the $\alpha_{1 \mathrm{~B}} \mathrm{~N}$-type $\mathrm{Ca}^{2+}$ channel derived from rat brain (Stea et al., 1999). Therefore, $\mathrm{Ca}^{2+}$ entry during an action potential can vary widely when examined across synapses and model preparations, even within the same family of $\mathrm{Ca}^{2+}$ channels. Despite these differences between synaptic preparations, our data are consistent with previous work at the frog neuromuscular junction that support the conclusion that the probability of $\mathrm{Ca}^{2+}$ channel opening during an action potential is very low. Using SPOFA, we were able to calculate the opening probability $p$ in our experimental preparation to be 0.24 , in agreement with previous estimates (Wachman et al., 2004). Furthermore, based on error estimates taken from extensive computer simulations, we show that the opening probability $p$ in our sample is likely somewhere in the range of $0.23-0.37$.

Our analysis approach assumes that all $\mathrm{Ca}^{2+}$ channels we sampled have the same probability of opening during an action potential. Thus, our calculation of the probability of opening during an action potential represents an average of the actual values for individual $\mathrm{Ca}^{2+}$ channels. It would be interesting to know how much variability in $p$ exists among individual active zone $\mathrm{Ca}^{2+}$ channels. Furthermore, it is likely that the probability of $\mathrm{Ca}^{2+}$ channel opening during an action potential can be modulated under different conditions (i.e., disease, altered synaptic activity, or pharmacologic treatment). In particular, this characteristic may be one that is modulated under conditions that trigger presynaptic mechanisms for transmitter release homeostasis (Wang et al., 2004; Frank et al., 2009).

\section{The number of $\mathrm{Ca}^{2+}$ channels within single active zones of the motor nerve terminal}

Despite previous work presenting evidence that the $\sim 200-250$ active zone intramembraneous particles observed in freezefracture active zone replicas include the voltage-gated $\mathrm{Ca}^{2+}$ channels that trigger vesicle release (Pumplin et al., 1981; Robitaille et al., 1990; Cohen et al., 1991) as well as $\mathrm{Ca}^{2+}$-activated potassium channels (Robitaille et al., 1993), it remains unknown how many of these active zone intramembraneous particles actually represent presynaptic $\mathrm{Ca}^{2+}$ channels. Furthermore, the stoichiometric relationship between presynaptic $\mathrm{Ca}^{2+}$ channels and the docked synaptic vesicles that line both sides of the active zone is unknown. In our experiments, an average of $\sim 18 \mathrm{Ca}^{2+}$ channels was reported by individual imaging pixels positioned over active zone regions of the nerve terminal. When we extended this single-pixel estimate to the number that would be expected to be present in an entire active zone (based on the optical resolution of the system) (Fig. 1), we estimate that each active zone has only $\sim 36$ voltage-gated $\mathrm{Ca}^{2+}$ channels along its entire length. Because we used the measured variance of fluorescence signal to calculate the number of $\mathrm{Ca}^{2+}$ channels in the active zone, other sources of variance (i.e., fluctuating $\mathrm{Ca}^{2+}$ flux through single channels) can influence this estimate. Based on error estimates for our SPOFA procedure obtained by extensive Monte Carlo simulations (see Results and Table 3), we predict that the number of voltage-gated $\mathrm{Ca}^{2+}$ channels per active zone is somewhere in the range of $34-53$.

Therefore, our results suggest that the number of active $\mathrm{Ca}^{2+}$ channels in an entire active zone constitutes only a small proportion ( $20 \%)$ of total intramembraneous particles (200-250) observed in freeze-fracture replicas. Because there are $\sim 25-40$ docked synaptic vesicles per active zone, our data raise the interesting possibility that each docked synaptic vesicle is tightly associated with only one voltage-gated $\mathrm{Ca}^{2+}$ channel (Fig. 8). Although this idea is consistent with the highly ordered $\mathrm{Ca}^{2+}$. regulated release machinery at the active zone (Harlow et al., 2001), we are unable to exclude the possibility of a heterogeneous distribution of $\mathrm{Ca}^{2+}$ channels along the active zone.

Of course, our analysis only estimates the number of functional presynaptic $\mathrm{Ca}^{2+}$ channels present in the active zone. There may be additional $\mathrm{Ca}^{2+}$ channels present that are "silent" as a result of some form of modulation such that they do not open during our experiments. A "silencing" of ion channels has been observed for potassium channels as a result of sumoylation (Rajan et al., 2005; Wilson and Rosas-Acosta, 2005), and something similar could occur with synaptic $\mathrm{Ca}^{2+}$ channels using any of a variety of potential mechanisms (Pun et al., 1986; Lipscombe et al., 1989; Faber et al., 1991; Voronin and Cherubini, 2004; Toselli et al., 2005). Variability in the number of active $\mathrm{Ca}^{2+}$ channels in an active zone then could be explained by some mechanism that underlies a "silent" to "functional" interconversion. Under this scenario, it is possible that some of the particles identified in freeze-fracture replicas of the frog neuromuscular junction may be silent $\mathrm{Ca}^{2+}$ channels.

\section{$\mathrm{The} \mathrm{Ca}^{2+}$ channel-vesicle release relationship}

The question of how many $\mathrm{Ca}^{2+}$ channels must open to trigger a single release event is particularly intriguing and contributes to our understanding of how $\mathrm{Ca}^{2+}$ regulates synaptic vesicle fusion in the nerve terminal. Historically, at some synapses, it has been proposed that many $\mathrm{Ca}^{2+}$ channels normally open to provide the $\mathrm{Ca}^{2+}$ trigger for vesicle fusion (Wheeler et al., 1994; Mintz et al., 1995; Borst and Sakmann, 1996; Wu et al., 1999), whereas at others it appears that one or only a few $\mathrm{Ca}^{2+}$ channels open to trigger each vesicle fusion event (Yoshikami et al., 1989; Augustine et al., 1991; Stanley, 1993; Brandt et al., 2005; Gentile and Stanley, 2005; Shahrezaei et al., 2006; Bucurenciu et al., 2010). Within individual active zones of the frog motor nerve terminal, our experimental data lead us to predict that an average of approximately nine $\mathrm{Ca}^{2+}$ channels open after an action potential (in which the range might be $8-18$ based on Monte Carlo simulations of predicted error), whereas vesicles are released with a probability of only $\sim 0.5$. Therefore, there appears to be a relatively low probability that these $\mathrm{Ca}^{2+}$ channel openings trigger vesicle fusion. One possible explanation is that each vesicle release event requires the summed $\mathrm{Ca}^{2+}$ flux through many open $\mathrm{Ca}^{2+}$ channels. However, previous studies at this synapse have suggested that $\mathrm{Ca}^{2+}$ flux through only one to two $\mathrm{Ca}^{2+}$ channels normally trigger transmitter release (Yoshikami et al., 1989; Shahrezaei et al., 2006). If only a few $\mathrm{Ca}^{2+}$ channel openings are required to trigger vesicle fusion, it is possible that these openings 
need to occur in close proximity to one another in the active zone to be effective at evoking transmitter release. The probability that two channel openings would occur in close proximity is low, which could therefore limit the occurrence of vesicle fusion from each active zone. Alternatively, each vesicle fusion event might be controlled predominately by one tightly associated $\mathrm{Ca}^{2+}$ channel positioned nearby but that $\mathrm{Ca}^{2+}$ flux through these single open channels might only trigger vesicle fusion with a low probability $(5-10 \%)$. In this case, the release probability within individual active zones would be determined by both the number of open $\mathrm{Ca}^{2+}$ channels and the probability that a single open $\mathrm{Ca}^{2+}$ channel triggers vesicle fusion.

\section{References}

Augustine GJ (1990) Regulation of transmitter release at the squid giant synapse by presynaptic delayed rectifier potassium current. J Physiol 431:343-364.

Augustine GJ, Adler EM, Charlton MP (1991) The calcium signal for transmitter secretion from presynaptic nerve terminals. Ann N Y Acad Sci 635:365-381.

Bertram R, Sherman A, Stanley EF (1996) Single-domain/bound calcium hypothesis of transmitter release and facilitation. J Neurophysiol 75:1919-1931.

Bischofberger J, Geiger JR, Jonas P (2002) Timing and efficacy of $\mathrm{Ca}^{2+}$ channel activation in hippocampal mossy fiber boutons. J Neurosci 22:10593-10602.

Borst JG, Sakmann B (1996) Calcium influx and transmitter release in a fast CNS synapse. Nature 383:431-434.

Borst JG, Sakmann B (1998) Calcium current during a single action potential in a large presynaptic terminal of the rat brainstem. J Physiol 506:143-157.

Bostock H, Sears TA, Sherratt RM (1981) The effects of 4-aminopyridine and tetraethylammonium ions on normal and demyelinated mammalian nerve fibres. J Physiol 313:301-315.

Brandt A, Khimich D, Moser T (2005) Few CaV1.3 channels regulate the exocytosis of a synaptic vesicle at the hair cell ribbon synapse. J Neurosci 25:11577-11585.

Braun M, Schmidt RF (1966) Potential changes recorded from the motor nerve terminal during its activation. Pflugers Arch 287:56-80.

Brenowitz SD, Regehr WG (2007) Reliability and heterogeneity of calcium signaling at single presynaptic boutons of cerebellar granule cells. J Neurosci 27:7888-7898.

Bucurenciu I, Bischofberger J, Jonas P (2010) A small number of open $\mathrm{Ca}^{2+}$ channels trigger transmitter release at a central GABAergic synapse. Nat Neurosci 13:19-21.

Carnevale NT, Hines ML (2006) The NEURON book. Cambridge, UK: Cambridge UP.

Church PJ, Stanley EF (1996) Single L-type calcium channel conductance with physiological levels of calcium in chick ciliary ganglion neurons. J Physiol 496:59-68.

Cohen MW, Jones OT, Angelides KJ (1991) Distribution of $\mathrm{Ca}^{2+}$ channels on frog motor nerve terminals revealed by fluorescent $\omega$-conotoxin. J Neurosci 11:1032-1039.

Del Castillo J, Katz B (1954) Quantal components of the end-plate potential. J Physiol 124:560-573.

Durant NN, Marshall IG (1980) The effects of 3,4-diaminopyridine on acetylcholine release at the frog neuromuscular junction. Eur J Pharmacol 67:201-208.

Faber DS, Lin JW, Korn H (1991) Silent synaptic connections and their modifiability. Ann NY Acad Sci 627:151-164.

Frank CA, Pielage J, Davis GW (2009) A presynaptic homeostatic signaling system composed of Eph receptor, ephexin, cdc42, and $\mathrm{Ca}_{\mathrm{v}} 2.1$ calcium channels. Neuron 61:556-569.

Frenguelli BG, Malinow R (1996) Fluctuations in intracellular calcium responses to action potentials in single en passage presynaptic boutons of layer v neurons in neocortical slices. Learn Mem 3:150-159.

Gentile L, Stanley EF (2005) A unified model of presynaptic release site gating by calcium channel domains. Eur J Neurosci 21:278-282.

Harlow ML, Ress D, Stoschek A, Marshall RM, McMahan UJ (2001) The architecture of active zone material at the frog's neuromuscular junction. Nature 409:479-484.
Heuser JE, Reese TS (1981) Structural changes after transmitter release at the frog neuromuscular junction. J Cell Biol 88:564-580.

Heuser JE, Reese TS, Dennis MJ, Jan Y, Jan L, Evans L (1979) Synaptic vesicle exocytosis captured by quick freezing and correlated with quantal transmitter release. J Cell Biol 81:275-300.

Hines ML, Carnevale NT (1997) The NEURON simulation environment. Neural Comput 9:1179-1209.

Jones SW, Marks TN (1989) Calcium currents in bullfrog sympathetic neurons. I. Activation kinetics and pharmacology. J Gen Physiol 94:151-167.

Katz B (1969) The release of neural transmitter substance. Liverpool, UK: Liverpool UP.

Katz B, Miledi R (1965) Propagation of electric activity in motor nerve terminals. Proc R Soc Lond B Biol Sci 161:453-482.

Katz B, Miledi R (1968) The effect of local blockage of motor nerve terminals. J Physiol 199:729-741.

Katz B, Miledi R (1979) Estimates of quantal content during "chemical potentiation" of transmitter release. Proc R Soc Lond B Biol Sci 205:369-378.

Kerr LM, Yoshikami D (1984) A venom peptide with a novel presynaptic blocking action. Nature 308:282-284.

Kerr RA, Bartol TM, Kaminsky B, Dittrich M, Chang JC, Baden SB, Sejnowski TJ, Stiles JR (2008) Fast Monte Carlo simulation methods for biological reaction-diffusion systems in solution and on surfaces. SIAM J Sci Comput 30:3126.

Kirsch GE, Narahashi T (1978) 3,4-diaminopyridine. A potent new potassium channel blocker. Biophys J 22:507-512.

Lin Z, Haus S, Edgerton J, Lipscombe D (1997) Identification of functionally distinct isoforms of the $\mathrm{N}$-type $\mathrm{Ca}^{2+}$ channel in rat sympathetic ganglia and brain. Neuron 18:153-166.

Lin Z, Lin Y, Schorge S, Pan JQ, Beierlein M, Lipscombe D (1999) Alternative splicing of a short cassette exon in alpha1B generates functionally distinct N-type calcium channels in central and peripheral neurons. J Neurosci 19:5322-5331.

Lipscombe D, Kongsamut S, Tsien RW (1989) Alpha-adrenergic inhibition of sympathetic neurotransmitter release mediated by modulation of N-type calcium-channel gating. Nature 340:639-642.

Mallart A (1984) Presynaptic currents in frog motor endings. Pflugers Arch 400:8-13.

Mintz IM, Sabatini BL, Regehr WG (1995) Calcium control of transmitter release at a cerebellar synapse. Neuron 15:675-688.

Pattillo JM, Yazejian B, DiGregorio DA, Vergara JL, Grinnell AD, Meriney SD (2001) Contribution of presynaptic calcium-activated potassium currents to transmitter release regulation in cultured Xenopus nerve-muscle synapses. Neuroscience 102:229-240.

Pawson PA, Grinnell AD, Wolowske B (1998) Quantitative freeze-fracture analysis of the frog neuromuscular junction synapse. I. Naturally occurring variability in active zone structure. J Neurocytol 27:361-377.

Poage RE, Meriney SD (2002) Presynaptic calcium influx, neurotransmitter release and neuromuscular disease. Physiol Behav 77:507-512.

Pumplin DW, Reese TS, Llinás R (1981) Are the presynaptic membrane particles the calcium channels? Proc Natl Acad Sci U S A 78:7210-7213.

Pun RY, Neale EA, Guthrie PB, Nelson PG (1986) Active and inactive central synapses in cell culture. J Neurophysiol 56:1242-1256.

Rajan S, Plant LD, Rabin ML, Butler MH, Goldstein SA (2005) Sumoylation silences the plasma membrane leak $\mathrm{K}^{+}$channel K2P1. Cell 121:37-47.

Rizzoli SO, Betz WJ (2005) Synaptic vesicle pools. Nat Rev Neurosci 6:57-69.

Robitaille R, Adler EM, Charlton MP (1990) Strategic location of calcium channels at transmitter release sites of frog neuromuscular synapses. Neuron 5:773-779.

Robitaille R, Garcia ML, Kaczorowski GJ, Charlton MP (1993) Functional colocalization of calcium and calcium-gated potassium channels in control of transmitter release. Neuron 11:645-655.

Sabatini BL, Regehr WG (1997) Control of neurotransmitter release by presynaptic waveform at the granule cell to Purkinje cell synapse. J Neurosci 17:3425-3435.

Sabatini BL, Svoboda K (2000) Analysis of calcium channels in single spines using optical fluctuation analysis. Nature 408:589-593.

Shahrezaei V, Cao A, Delaney KR (2006) $\mathrm{Ca}^{2+}$ from one or two channels controls fusion of a single vesicle at the frog neuromuscular junction. J Neurosci 26:13240-13249. 
Stanley EF (1993) Single calcium channels and acetylcholine release at a presynaptic nerve terminal. Neuron 11:1007-1011.

Stea A, Dubel SJ, Snutch TP (1999) Alpha 1B N-type calcium channel isoforms with distinct biophysical properties. Ann NY Acad Sci 868:118-130.

Stiles JR, Bartol TM (2001) Monte Carlo methods for simulating realistic synaptic microphysiology using MCell. In: Computational neuroscience: realistic modeling for experimentalists (De Schutter E, ed), pp 87-127. Boca Raton, FL: CRC

Stiles JR, Van Helden D, Bartol TM Jr, Salpeter EE, Salpeter MM (1996) Miniature endplate current rise times $<100 \mu$ s from improved dual recordings can be modeled with passive acetylcholine diffusion from a synaptic vesicle. Proc Natl Acad Sci U S A 93:5747-5752.

Stocker JW, Nadasdi L, Aldrich RW, Tsien RW (1997) Preferential interaction of omega-conotoxins with inactivated $\mathrm{N}$-type $\mathrm{Ca}^{2+}$ channels. J Neurosci 17:3002-3013.

Thomsen RH, Wilson DF (1983) Effects of 4-aminopyridine and 3,4 diaminopyridine on transmitter release at the neuromuscular junction. J Pharmacol Exp Ther 227:260-265.

Toselli M, Biella G, Taglietti V, Cazzaniga E, Parenti M (2005) Caveolin-1 expression and membrane cholesterol content modulate $\mathrm{N}$-type calcium channel activity in NG108-15 cells. Biophys J 89:2443-2457.

Voronin LL, Cherubini E (2004) "Deaf, mute and whispering" silent synapses: their role in synaptic plasticity. J Physiol 557:3-12.
Wachman ES, Poage RE, Stiles JR, Farkas DL, Meriney SD (2004) Spatial distribution of calcium entry evoked by single action potentials within the presynaptic active zone. J Neurosci 24:2877-2885.

Wang X, Engisch KL, Li Y, Pinter MJ, Cope TC, Rich MM (2004) Decreased synaptic activity shifts the calcium dependence of release at the mammalian neuromuscular junction in vivo. J Neurosci 24:1068710692.

Wheeler DB, Randall A, Tsien RW (1994) Roles of N-type and Q-type Ca ${ }^{2+}$ channels in supporting hippocampal synaptic transmission. Science 264:107-111.

Wilson VG, Rosas-Acosta G (2005) Wrestling with SUMO in a new arena. Sci STKE 290:32.

Wood SJ, Slater CR (2001) Safety factor at the neuromuscular junction. Prog Neurobiol 64:393-429.

Wu LG, Westenbroek RE, Borst JG, Catterall WA, Sakmann B (1999) Calcium channel types with distinct presynaptic localization couple differentially to transmitter release in single calyx-type synapses. J Neurosci 19:726-736.

Yoshikami D, Bagabaldo Z, Olivera BM (1989) The inhibitory effects of omega-conotoxins on Ca channels and synapses. Ann NY Acad Sci 560:230-248

Zhai RG, Bellen HJ (2004) The architecture of the active zone in the presynaptic nerve terminal. Physiology 19:262-270. 